\title{
Large deviation spectra based on wavelet leaders
}

\author{
Françoise Bastin, Céline Esser and Stéphane Jaffard
}

\begin{abstract}
We introduce a quantity that encapsulates the information given by the distribution at each scale of the wavelet leaders of a function, and which is independent of the chosen wavelet basis. This leads naturally to the definition of a new multifractal formalism which allows to detect non-concave multifractal spectra.
\end{abstract}

\section{Introduction}

Multifractal analysis is concerned with the study of everywhere irregular signals whose pointwise regularity can change widely from one point to another. For such data, it does not make sense to determine the pointwise regularity, and it is more relevant to determine their multifractal spectrum, which is the Hausdorff dimension of the set of points which have a given Hölder exponent. More precisely, let $\alpha$ be a non-negative real number and $x_{0} \in \mathbb{R}$. A locally bounded function $f: \mathbb{R} \rightarrow \mathbb{R}$ belongs to $C^{\alpha}\left(x_{0}\right)$ if there exist a polynomial $P$ of degree less than $\alpha$ and a constant $C>0$ such that

$$
|f(x)-P(x)| \leq C\left|x-x_{0}\right|^{\alpha}
$$

for every $x$ in a neighborhood of $x_{0}$. The Hölder exponent $h_{f}\left(x_{0}\right)$ of $f$ at $x_{0}$ is the supremum of all values of $\alpha$ such that $f \in C^{\alpha}\left(x_{0}\right)$. Points with a given Hölder exponent can be located on fractal sets, in which case their Hausdorff dimension is a pertinent quantity to determine. If $B_{h}$ denotes the set of points $x \in \mathbb{R}$ for which $h_{f}(x)=h$, the multifractal spectrum of $f$ is the function

$$
d_{f}: h \mapsto \operatorname{dim}_{\mathcal{H}}\left(B_{h}\right) .
$$

Note that $h_{f}\left(x_{0}\right)$ can be infinite, so that $d_{f}$ is defined on $[0,+\infty]$. Furthermore, one uses the standard convention $\operatorname{dim}(\emptyset)=-\infty$ so that $d_{f}$ takes values in $[0,1] \cup\{-\infty\}$.

The Hölder exponent and hence the multifractal spectrum of many mathematical functions or stochastic models has been directly determined from their definition; usually their Hölder exponent is extremely erratic, see e.g. [8, 20]. Therefore,

Mathematics Subject Classification (2010): 42C40, 46E36, $26 \mathrm{~A} 16$.

Keywords: Large deviation spectra, $S^{\nu}$ spaces, multifractal analysis, profile spaces, wavelet leaders. 
for real-life signals, the Hölder exponent is also expected to be very erratic and its numerical determination is not feasible. Thus, one cannot expect to have direct access to their spectrum. In such cases, one has to find an indirect way to estimate it. A multifractal formalism is a formula which is expected to yield the spectrum of a function from "global" quantities which are numerically computable. The seminal work of G. Parisi and U. Frisch [36] led to a first formula, which related the spectrum and the scaling function $\eta_{f}(p)$ of the signal which (using a loose formulation) is defined by

$$
\int|f(x+h)-f(x)|^{p} d x \sim h^{\eta_{f}(p)} \quad \text { when } h \rightarrow 0 .
$$

One expected that the Legendre transform of $\eta$

$$
\mathcal{L}_{f}(H):=\inf _{p \in \mathbb{R}}\left(1+H p-\eta_{f}(p)\right)
$$

would yield the multifractal spectrum of $f$. This expectation was only partly met: For large classes of functions and stochastic models that have a concave (increasing and then decreasing) spectrum, this heuristic has been shown to correctly yield the increasing part of the spectrum (where the infimum in (0.2) is attained for $p>$ 0 ). Furthermore, a function space interpretation can be given to the multifractal formalism. This interpretation leads to generic results of validity: For $p>0,(0.1)$ is interpreted as stating that $f$ belongs to an intersection of Besov spaces, see [21]. The multifractal formalism yields the increasing part of the spectrum for a subset of the function space considered which is a comeager set from a topological point of view or a prevalent set from a probabilistic point of view, see [18, 21].

However, this formalism proves problematic when $p<0$ (which corresponds to the decreasing part of the spectrum in the Legendre transform formula) because, in the neighborhood of points where the increments of $f$ vanish, the estimation of the integral (0.1) becomes highly unstable or even divergent, leading to meaningless values for the scaling function, and, ultimately, to the failure of the multifractal formalism even for standard models (see [25] where this phenomenon is analyzed in details on the example of Brownian motion).

Several approaches have been proposed in order to "renormalize" $(0.1)$ when $p$ is negative. A first way, proposed by A. Arneodo, E. Bacry and J.-F. Muzy, consists in replacing increments by a continuous wavelet transform and the integral by a sum taken at the points of its local maxima. On the numerical side, the wavelet transform maxima method proved extremely powerful, yielding the correct full spectra (when concave) for large classes of synthetic signals, see [3, 34, 35]. However the main difficulty of this method is that mathematical results backing it are extremely scarce (see however $[3,19,30]$ ) and far from yielding a proof of its validity, even for standard models such as e.g. Brownian motion. This drawback motivated the introduction of an alternative method which is based on a similar idea, see [24, 26]: One uses an orthonormal wavelet basis, and the sum does not bear on wavelet coefficients but on local suprema of these coefficients (called wavelet leaders, see (0.4) below). Three types of mathematical results have been 
obtained concerning the validity of this multifractal formalism, see $[1,2,23]$ and references therein:

- It is proved for specific deterministic functions or for classes of stochastic models (multiplicative cascades, Lévy processes without Brownian component, random wavelet series,...) where it allows to recover the increasing and the decreasing part of the spectrum.

- It is shown to yield an upper bound of the multifractal spectrum of any (uniformly Hölder) function.

- The part which has a function space interpretation $(p>0$ in the cases we mentioned, yielding the increasing part of the spectrum) is proved to hold for a generic subset of the corresponding function space.

However, this method still presents two main drawbacks:

- The scaling function approach can encapsulate a "function space - type" information only for $p>0$, so that generic results can only concern the increasing part of spectra.

- The Legendre transform, by construction, can only yield a concave output, so that this multifractal formalism necessarily fails for functions with a nonconcave spectrum.

The first drawback is intrinsic to the notions of genericity which are used, but the second one can be partly turned by using a method which differs from the scaling function/ Legendre transform technique; the use of function spaces which differ from Besov spaces and are based on large deviating estimates of the repartition of wavelet coefficients (the so-called $\mathcal{S}^{\nu}$ spaces [5]) allows to deal with non-concave spectra. Our purpose in this paper is to combine this approach with the use of wavelet leaders instead of wavelet coefficients and propose new spectra derived from large deviations based on statistics of wavelet leaders. We will show that, indeed, this method allows access to both the increasing and decreasing envelopes of non-concave multifractal spectra. Note that typical examples of stochastic models with non-concave spectra are given by Lévy processes with a Brownian component, see [20]. They can also easily be obtained by juxtaposing signals with different spectra, a situation commonly met in image processing, because of the occlusion phenomenon (a natural image usually is a juxtaposition of different textures with different spectra).

In order to be more specific we start by giving some precisions about $\mathcal{S}^{\nu}$ spaces and we introduce some notations. We will only consider one-variable functions (it is straightforward to generalize what follows in the case of dimension $d>1$ ). Since we are interested in local properties, we work with periodic functions. Let us consider a mother wavelet $\psi$ in the Schwartz class, such as constructed in [29]. Together with the constant function $\varphi(x):=1$, the periodic functions

$$
\psi_{j, k}(x):=\sum_{l \in \mathbb{Z}} \psi\left(2^{j}(x-l)-k\right), \quad j \in \mathbb{N}_{0}, k \in\left\{0, \ldots, 2^{j}-1\right\}
$$


form an orthogonal basis of functions of period 1 in $L^{2}(\mathbb{T})$, where $\mathbb{T}=\mathbb{R} / \mathbb{Z}$. We use the notations $\psi_{\lambda}$ to denote the wavelet $\psi_{j, k}$, where $\lambda$ is the dyadic interval

$$
\lambda=\lambda(j, k)=\left[\frac{k}{2^{j}}, \frac{k+1}{2^{j}}\right), \quad j \in \mathbb{N}_{0}, k \in\left\{0, \ldots, 2^{j}-1\right\}
$$

(the interval $\lambda$ gives an indication concerning the position and scale of the corresponding wavelet). We denote by $\Lambda$ the set of all dyadic intervals included in $[0,1)$; and if $j$ is a non-negative integer, we denote by $\Lambda_{j}$ the set of all dyadic intervals of $[0,1)$ of size $2^{-j}$. Using an $L^{\infty}$-normalization, the wavelet coefficients of a periodic function $f$ are

$$
\forall \lambda \in \Lambda_{j}, \quad c_{\lambda}=2^{j} \int_{0}^{1} f(x) \psi_{\lambda}(x) d x .
$$

The wavelet profile of $f$ is defined by

$$
\nu_{f}(\alpha):=\lim _{\varepsilon \rightarrow 0^{+}} \limsup _{j \rightarrow+\infty} \frac{\log \#\left\{\lambda \in \Lambda_{j}:\left|c_{\lambda}\right| \geq C 2^{-(\alpha+\varepsilon) j}\right\}}{\log 2^{j}}
$$

for all $\alpha \in \mathbb{R}$, where $\# A$ denotes the cardinality of $A$. This definition formalizes the idea that there are approximately $2^{\nu_{f}(\alpha) j}$ coefficients in modulus greater than $2^{-\alpha j}$. Note that, in the setting of probability measures similar quantities are considered in [32].

The function $\nu_{f}$ is non decreasing, right-continuous and takes values in $\{-\infty\} \cup$ $[0,1]$. The wavelet profile contains the maximal information which can be obtained from the distribution of wavelet coefficients of $f$ and which does not depend on the chosen wavelet basis, see [22]; furthermore, if $\nu_{f}$ is not concave, then it contains strictly more information than the knowledge of the Besov spaces to which $f$ belongs (which only allows to recover the concave hull of $\nu_{f}$ ).

In order to obtain a framework to model this type of information, the following definition was proposed in [22]: Given a function $\nu$ defined on $\mathbb{R}$, non decreasing, right-continuous and taking values in $\{-\infty\} \cup[0,1]$, a function $f$ belongs to the space $\mathcal{S}^{\nu}$ if its wavelet profile satisfies

$$
\nu_{f}(\alpha) \leq \nu(\alpha), \quad \forall \alpha \in \mathbb{R} .
$$

As mentioned previously, those spaces allow to state a new multifractal formalism which is fitted to non concave spectra. It is based on the estimation of the multifractal spectrum of any function of $\mathcal{S}^{\nu}$ by the increasing function

$$
d^{\nu}(h)= \begin{cases}h \cdot \sup _{h^{\prime} \in(0, h]} \frac{\nu\left(h^{\prime}\right)}{h^{\prime}} & \text { if } h \leq h_{\max } \\ 1 & \text { otherwise }\end{cases}
$$

where

$$
h_{\max }:=\inf _{h \geq \alpha_{\min }} \frac{h}{\nu(h)} \quad \text { and } \quad \alpha_{\min }:=\inf \{\alpha: \nu(\alpha) \geq 0\}
$$




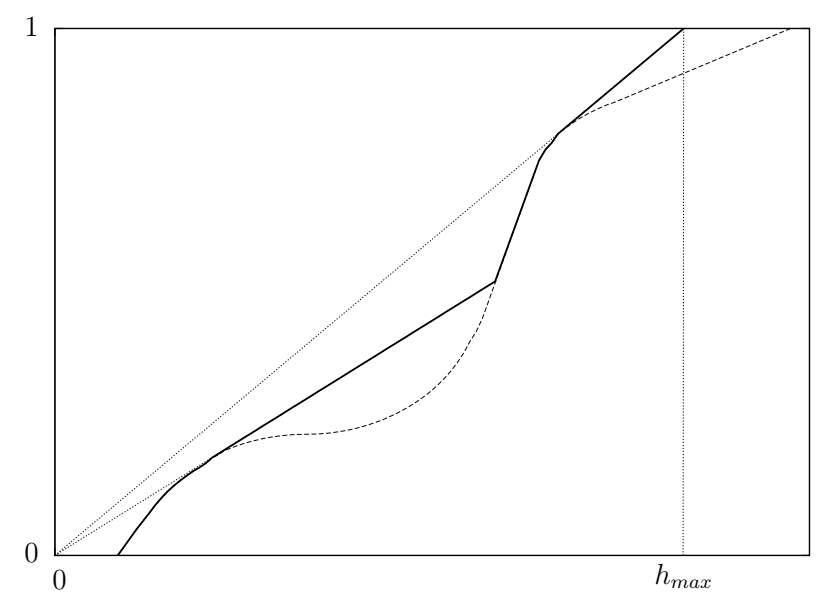

Figure 1: Example of $\nu(---)$ and $d^{\nu}(-)$

This formalism yields an upper bound for the spectrum of all uniformly Hölder function (i.e. for functions that belong to a Hölder space $C^{r}(\mathbb{T})$ for an $r>0$, see Section 1), and its validity has been proved for random wavelet series, and in generic settings, see $[4,5,7]$. Moreover, it has been implemented and tested on several examples [27].

Recall that the initial multifractal formalism based on increments fails to yield the decreasing part of spectra; the same problem shows up when using wavelet coefficients for the computation of the scaling function, i.e. if one replaces the integral in (0.1) by the quantity

$$
2^{-j} \sum_{\lambda \in \Lambda_{j}}\left|c_{\lambda}\right|^{p}
$$

see [25]. More accurate results are obtained when, rather than using directly wavelet coefficients, one relies on alternative quantities, namely wavelet leaders $d_{\lambda}$ which are defined as follows: Let $3 \lambda$ denote the interval with the same center as $\lambda$ but three times larger; then

$$
d_{\lambda}=\sup _{\lambda^{\prime} \subset 3 \lambda}\left|c_{\lambda^{\prime}}\right|, \quad \forall \lambda \in \Lambda
$$

(this supremum is finite as soon as $f$ is locally bounded) see [26]. This improvement is due to specificities of the wavelet leaders: Given a scale, wavelet leaders take into account a specific family of coefficients of smaller scales and located at the same place. Thus, heuristically, if a wavelet leader is close to 0 , this means that all these wavelet coefficients are close to 0 , which is much more exceptional that just one. This explains why the computation of scaling functions for negative $p \mathrm{~s}$, 
which amounts to replace (0.3) by

$$
2^{-j} \sum_{\lambda \in \Lambda_{j}}\left|d_{\lambda}\right|^{p}
$$

leads to more stable quantities. Additionally, wavelet leaders have adequate theoretical properties for the construction of a multifractal formalism, since they are directly related with the Hölder exponent, which can be recovered from wavelet leaders by local log-log plot regressions, see [23]. A multifractal formalism, based on a scaling function derived from (0.3) allows to estimate both the increasing and decreasing parts of concave spectra, see $[24,26]$.

Of course, the question of extending the study of $\mathcal{S}^{\nu}$ spaces in the context of the wavelet leaders was raised. These spaces, called profile spaces $\mathcal{L}^{\nu}$, (and which no longer are vector spaces) yield a new multifractal formalism which allows to detect non-concave and decreasing spectra.

This article is structured as follows: In Section 1, we introduce a large deviation spectrum based on the wavelet leaders of a function. The relevance of this spectrum comes from the fact that it gives an upper bound for the multifractal spectrum of the corresponding function. In Section 2, we show a drawback of this definition: It may depend on the wavelet basis chosen to compute it. This is why we derive in Section 3 another quantity based on the wavelet leaders which still yield an upper bound for the spectrum (which may however be less sharp than the one obtained in Section 1). It is proved to be independent of the chosen wavelet basis in Section 4. It allows to propose a new multifractal formalism and to define in Section 5 the profile spaces $\mathcal{L}^{\nu}$. In Section 6 , we illustrate this formalism on examples derived from the simplest multifractal cascade models. Let us already mention that this method is studied in practice and compared with the other wavelet-based formalisms in $[1,16]$. Topological properties of the new spaces $\mathcal{L}^{\nu}$ and the comparison with the $\mathcal{S}^{\nu}$ spaces have also already been investigated in [11].

\section{Upper bound for the multifractal spectrum}

In this section, we define a large deviation spectrum based on the wavelet leaders of a function in a given wavelet basis, and we will show that this quantity yields an upper bound for the multifractal function of the signal. A similar approach has been followed in [9] using oscillations of the function (i.e. the difference between the supremum and the infimum of the function on an interval) instead of wavelet leaders. The advantage of wavelet leaders is that they allow to deal with Hölder exponents larger than 1 . Note that the first ideas of deriving large deviation estimates for models that would turn out later to be multifractal can be traced back to the seminal note of B. Mandelbrot on cascade models for the modeling of turbulence [31].

The following notations will be used. The set of positive natural numbers is denoted by $\mathbb{N}, \mathbb{N}_{0}=\{0\} \cup \mathbb{N}$ and $\Omega$ is the set of complex sequences 
$\vec{c}=\left(c_{j, k}\right)_{j \in \mathbb{N}_{0}, k \in\left\{0, \ldots, 2^{j}-1\right\}}$. It will usually be interpreted as the sequence of wavelet coefficients of a periodic function $f$ in a given wavelet basis.

For every $r>0$ such that $r \notin \mathbb{N}$, we denote by $C^{r}(\mathbb{T})$ the space of 1-periodic functions which are Hölder continuous of order $r$. The wavelet characterization of Hölder spaces allows to identify this space with the subspace of $\Omega$ composed of sequences satisfying

$$
\sup _{j \in \mathbb{N}_{0}} \sup _{k \in\left\{0, \ldots, 2^{j}-1\right\}} 2^{r j}\left|c_{j, k}\right|<+\infty,
$$

see [33]. When $r \in \mathbb{N}_{0}$, we will also denote by $C^{r}(\mathbb{T})$ the space of functions, or wavelet coefficients, satisfying this condition (see [33] for a interpretation of this condition using the Zygmund class). A function (or a wavelet sequence) is uniformly Hölder if it belongs to a space $C^{r}(\mathbb{T})$, for an $r>0$. Note that, if $r>0$, this is a stronger requirement than continuity. As soon as $\vec{c} \in C^{0}(\mathbb{T}$ ) (which is verified if $f \in L^{\infty}$ ), the wavelet leaders are well defined and finite, and we denote the corresponding collection by $\vec{d}=\left(d_{j, k}\right)_{j \in \mathbb{N}_{0}, k \in\left\{0, \ldots, 2^{j}-1\right\}}=\left(d_{\lambda}\right)_{\lambda \in \Lambda}$.

Definition 1.1. Let $\vec{c} \in C^{0}(\mathbb{T})$. The wavelet leaders density $\rho_{\vec{c}}$ of $\vec{c}$ is defined for every $\alpha \geq 0$ by

$$
\rho_{\vec{c}}(\alpha):=\lim _{\varepsilon \rightarrow 0^{+}} \limsup _{j \rightarrow+\infty} \frac{\log \#\left\{\lambda \in \Lambda_{j}: 2^{-(\alpha+\varepsilon) j} \leq d_{\lambda}<2^{-(\alpha-\varepsilon) j}\right\}}{\log 2^{j}}
$$

and for $\alpha=+\infty$ by

$$
\rho_{\vec{c}}(+\infty):=\lim _{A \rightarrow+\infty} \liminf _{j \rightarrow+\infty} \frac{\log \#\left\{\lambda \in \Lambda_{j}: d_{\lambda} \leq 2^{-A j}\right\}}{\log 2^{j}}
$$

From typical properties of large deviation spectra, we get that the wavelet leaders density is upper semi-continuous on $[0,+\infty)$ and its maximum on $[0,+\infty]$ is equal to 1 (see [7] for example).

In this paper, $\vec{c}$ will usually denote the sequence of wavelet coefficients of a uniformly Hölder function $f$ in a fixed wavelet basis. Moreover, whenever the context is clear, we will write $\rho$ instead of $\rho_{\vec{c}}$ to make the notation less cluttered. In order to prove that the wavelet leaders density has the announced property, let us first introduce some notations. For every $x_{0} \in \mathbb{T}$, we denote by $\lambda_{j}\left(x_{0}\right)$ the dyadic interval of size $2^{-j}$ containing $x_{0}$ and $d_{j}\left(x_{0}\right)=\sup _{\lambda^{\prime} \subset 3 \lambda_{j}\left(x_{0}\right)}\left|c_{\lambda^{\prime}}\right|$. For every $\alpha \in \mathbb{R}$, we set

$$
F^{j}(\alpha)=\left\{k \in\left\{0, \ldots, 2^{j}-1\right\}: d_{j, k} \geq 2^{-\alpha j}\right\} \text { and } E^{j}(\alpha)=\bigcup_{k \in F^{j}(\alpha)} \lambda_{j, k} .
$$

We also define

$$
E(\alpha)=\limsup _{j \rightarrow+\infty} E^{j}(\alpha)=\bigcap_{j \in \mathbb{N}_{0}} \bigcup_{m \geq j} E^{m}(\alpha) .
$$

Remark that, since $f$ is uniformly Hölder, there exist $\alpha_{0}>0$ and $C>0$ such that

$$
\forall j \in \mathbb{N}, k \in\left\{0, \ldots, 2^{j}-1\right\}, \quad\left|c_{j, k}\right| \leq C 2^{-\alpha_{0} j} .
$$


Therefore, $E(\alpha)=\emptyset$ if $\alpha<\alpha_{0}$.

The following result from [23] is a characterization of the Hölder exponent of a uniformly Hölder function using its wavelet leaders.

Proposition 1.2. If $f$ is a uniformly Hölder function, then

$$
h_{f}\left(x_{0}\right)=\liminf _{j \rightarrow+\infty} \frac{\log d_{j}\left(x_{0}\right)}{\log 2^{-j}} .
$$

Lemma 1.3. Let $f$ be a uniformly Hölder function and let $\alpha \geq 0$.

1. If $x_{0} \in E(\alpha)$, then $h_{f}\left(x_{0}\right) \leq \alpha$.

2. If $h_{f}\left(x_{0}\right)<\alpha$, then $x_{0} \in E(\alpha)$.

Proof. 1. Let us assume that $x_{0} \in E(\alpha)$. Then for every $j$, there exist $m_{j} \geq j$ and $k_{j} \in F^{m_{j}}(\alpha)$ such that $x_{0} \in \lambda_{m_{j}, k_{j}}$. This means that $d_{m_{j}}\left(x_{0}\right)=d_{m_{j}, k_{j}} \geq 2^{-\alpha m_{j}}$. It follows that

$$
h_{f}\left(x_{0}\right)=\liminf _{j \rightarrow+\infty} \frac{\log d_{j}\left(x_{0}\right)}{\log 2^{-j}} \leq \lim _{j \rightarrow+\infty} \frac{\log d_{m_{j}, k_{j}}}{\log 2^{-m_{j}}} \leq \lim _{j \rightarrow+\infty} \frac{\log 2^{-\alpha m_{j}}}{\log 2^{-m_{j}}}=\alpha .
$$

2. Let us assume that $h_{f}\left(x_{0}\right)<\alpha$. Then, there exists an increasing sequence $\left(j_{n}\right)_{n \in \mathbb{N}}$ of natural numbers such that

$$
\frac{\log d_{j_{n}}\left(x_{0}\right)}{\log 2^{-j_{n}}}<\alpha
$$

Consequently, $d_{j_{n}}\left(x_{0}\right)>2^{-\alpha j_{n}}$ and $x_{0} \in E^{j_{n}}(\alpha)$.

Lemma 1.4. If $f$ is a uniformly Hölder function, then for every $h \geq 0$

$$
\left\{x_{0}: h_{f}\left(x_{0}\right)=h\right\}=\bigcap_{\varepsilon>0} E(h+\varepsilon) \backslash E(h-\varepsilon) .
$$

Proof. The result is obtained directly from Lemma 1.3.

Theorem 1.5. If $f$ is a uniformly Hölder function, then its multifractal spectrum satisfies

$$
d_{f}(h) \leq \rho(h), \quad \forall h \in[0,+\infty] .
$$

Proof. We first consider the case where $h \in[0,+\infty)$. Because of Lemma 1.4, we have to show that

$$
\operatorname{dim}_{\mathcal{H}}\left(\bigcap_{\varepsilon>0} E(h+\varepsilon) \backslash E(h-\varepsilon)\right) \leq \rho(h) .
$$

Let us consider $\delta>0$. From the definition of the wavelet leaders density $\rho$, there exists $\varepsilon_{0}>0$ and $j_{0} \in \mathbb{N}_{0}$ such that

$$
\#\left\{\lambda \in \Lambda_{j}: 2^{-(h+\varepsilon) j} \leq d_{\lambda}<2^{-(h-\varepsilon) j}\right\} \leq 2^{(\rho(h)+\delta) j}, \quad \forall j \geq j_{0} .
$$


If we set

$$
E_{\varepsilon_{0}}^{j}(h)=E^{j}\left(h+\varepsilon_{0}\right) \backslash E^{j}\left(h-\varepsilon_{0}\right), \quad \forall j \in \mathbb{N}_{0},
$$

then

$$
E\left(h+\varepsilon_{0}\right) \backslash E\left(h-\varepsilon_{0}\right) \subseteq \bigcap_{J \in \mathbb{N}_{0}} \bigcup_{j \geq J} E_{\varepsilon_{0}}^{j}(h)
$$

Let us show that

$$
\mathcal{H}^{s}\left(\bigcap_{J \in \mathbb{N}_{0}} \bigcup_{j \geq J} E_{\varepsilon_{0}}^{j}(h)\right)<+\infty
$$

where $s=\rho(h)+2 \delta$. Remark that for every $j \in \mathbb{N}$, the set $E_{\varepsilon_{0}}^{j}(h)$ is covered by $\#\left\{\lambda \in \Lambda_{j}: 2^{-\left(h+\varepsilon_{0}\right) j} \leq d_{\lambda}<2^{-\left(h-\varepsilon_{0}\right) j}\right\}$ intervals of length $2^{-j}$. For every $\eta>0$, there is $J(\eta) \geq j_{0}$ such that $2^{-j} \leq \eta$ if $j \geq J(\eta)$. Then, we have

$$
\begin{aligned}
\mathcal{H}_{\eta}^{s}\left(\bigcap_{J \in \mathbb{N}_{0}} \bigcup_{j \geq J} E_{\varepsilon_{0}}^{j}(h)\right) & \leq \mathcal{H}_{\eta}^{s}\left(\bigcup_{j \geq J(\eta)} E_{\varepsilon_{0}}^{j}(h)\right) \\
& \leq \sum_{j \geq J(\eta)}\left(\#\left\{\lambda \in \Lambda_{j}: 2^{-(h+\varepsilon) j} \leq d_{\lambda}<2^{-(h-\varepsilon) j}\right\}\right) 2^{-s j} \\
& \leq \sum_{j \geq J(\eta)} 2^{(\rho(h)+\delta) j} 2^{-s j} \leq \sum_{j \in \mathbb{N}_{0}} 2^{-\delta j}<+\infty
\end{aligned}
$$

Consequently,

$$
\mathcal{H}^{s}\left(\bigcap_{J \in \mathbb{N}_{0}} \bigcup_{j \geq J} E_{\varepsilon_{0}}^{j}(h)\right)=\lim _{\eta \rightarrow 0^{+}} \mathcal{H}_{\eta}^{s}\left(\bigcap_{J \in \mathbb{N}_{0}} \bigcup_{j \geq J} E_{\varepsilon_{0}}^{j}(h)\right) \leq \sum_{j \in \mathbb{N}_{0}} 2^{-\delta j}<+\infty
$$

and it follows that

$$
\operatorname{dim}_{\mathcal{H}}\left(\bigcap_{\varepsilon>0} E(h+\varepsilon) \backslash E(h-\varepsilon)\right) \leq \operatorname{dim}_{\mathcal{H}}\left(\bigcap_{J \in \mathbb{N}_{0}} \bigcup_{j \geq J} E_{\varepsilon_{0}}^{j}(h)\right) \leq s=\rho(h)+2 \delta .
$$

Since $\delta>0$ is arbitrary, we finally get

$$
\operatorname{dim}_{\mathcal{H}}\left(\bigcap_{\varepsilon>0} E(h+\varepsilon) \backslash E(h-\varepsilon)\right) \leq \rho(h)
$$

which leads to the conclusion.

We now consider the case $h=+\infty$. If we set

$$
B_{A}(j)=\bigcup_{\lambda \in \Lambda_{j}: d_{\lambda} \leq 2^{-A J}} \lambda,
$$

then

$$
f \in C^{\infty}\left(x_{0}\right) \Longleftrightarrow \forall A, \quad \forall j \text { large enough } \quad x_{0} \in B_{A}(j) .
$$

Thus, for any $A$, the set $\lim \inf B_{A}(j)$ supplies a covering of $\left\{x_{0}: h_{f}\left(x_{0}\right)=+\infty\right\}$. The result follows as previously from the definition of $\rho(+\infty)$. 
One drawback when dealing with wavelet leaders is that the suprema corresponding to two neighbors dyadic intervals overlap; for instance, in a probabilistic framework, this will create correlations between wavelet leaders, even if they didn't exist between wavelet coefficients. Therefore it is natural to wonder if the developments that we pursued could be developed in a simpler framework where wavelet leaders are replaced by restricted wavelet leaders defined by

$$
e_{\lambda}=\sup _{\lambda^{\prime} \subset \lambda}\left|c_{\lambda^{\prime}}\right|, \quad \lambda \in \Lambda \text {. }
$$

As before, we can consider the function

$$
\rho_{\vec{c}}^{\operatorname{restr}}(\alpha)=\lim _{\varepsilon \rightarrow 0^{+}} \limsup _{j \rightarrow+\infty} \frac{\log \#\left\{\lambda \in \Lambda_{j}: 2^{-(\alpha+\varepsilon) j} \leq e_{\lambda}<2^{-(\alpha-\varepsilon) j}\right\}}{\log 2^{j}} .
$$

Let us show that $\rho_{\vec{c}} \leq \rho_{\vec{c}}^{\text {restr }}$ but that these functions do not necessarily coincide, i.e. there exists $\vec{c}$ such that $\rho_{\vec{c}}^{\text {restr }} \not \leq \rho_{\vec{c}}$.

First, remark that $d_{\lambda}=\max \left\{e_{\mu}: \mu \in N(\lambda)\right\}$ where $N(\lambda)$ denotes the set of the 3 "neighbors" of $\lambda$ in $\Lambda_{j}$ (i.e., the dyadic intervals of length $2^{-j}$, whose boundary intersects the boundary of $\lambda$ ). Therefore,

$\#\left\{\lambda \in \Lambda_{j}: 2^{-(\alpha+\varepsilon) j} \leq d_{\lambda}<2^{-(\alpha-\varepsilon) j}\right\} \leq 3 \#\left\{\lambda \in \Lambda_{j}: 2^{-(\alpha+\varepsilon) j} \leq e_{\lambda}<2^{-(\alpha-\varepsilon) j}\right\}$

and it follows that for any sequence $\vec{c}$, we have $\rho_{\vec{c}} \leq \rho_{\vec{c}}^{\text {restr }}$.

Let us now check that these two quantities can differ. Consider the Cantor set of ratio $\frac{1}{4}$ : We start with the interval [0,1], and, at each step in the standard Cantor set construction, we keep the two outer dyadic intervals whose length is $\frac{1}{4}$ times the length of the parent interval. We denote $C_{n}\left(\frac{1}{4}\right)$ the subset of $[0,1]$ obtained at step $n$ and we denote the Cantor set by

$$
C\left(\frac{1}{4}\right)=\bigcap_{n \in \mathbb{N}} C_{n}\left(\frac{1}{4}\right)
$$

Note that the dyadic intervals $\lambda(j, k)$ that show up in the construction (we will call them the "fundamental intervals") possess an index $j$ that is even.

We now pick a wavelet coefficient sequence as follows: Let $0<\gamma<\alpha$.

- Let $j$ be even. If $\lambda(j, k)$ is a fundamental interval, we set $c_{j, k}=2^{-\gamma j}$. If $\lambda(j, k)$ is a subinterval of a fundamental interval of the generation $j-2$ (we will call them the "secondary intervals"), then we set $c_{j, k}=2^{-\alpha j}$, and otherwise, we set $c_{j, k}=0$.

- Let now $j$ be odd. If $\lambda(j, k)$ is a subinterval of a fundamental interval of the generation $j-1$, we set $c_{j, k}=2^{-\gamma j}$. Otherwise, we set $c_{j, k}=0$.

One easily checks that all wavelet leaders are either of size $2^{-\gamma j}$ or 0 , while restricted wavelet leaders associated to a secondary interval are of size $2^{-\alpha j}$ (indeed, this is the size of the corresponding wavelet coefficients, and all wavelet coefficients associated to proper subintervals vanish). Consequently, $\rho_{\vec{c}}^{\text {restr }}(\alpha) \neq \rho_{\vec{c}}(\alpha)$. 
Remark 1.6. From Theorem 1.5, if $f$ is uniform Hölder, we have $d_{f}(h) \leq \rho_{\vec{c}}(h)$ for every $h \geq 0$. So, we also have $d_{f}(h) \leq \rho_{\vec{c}}^{\text {restr }}(h)$ for every $h \geq 0$.

The example that we just exposed shows that the upper bound of the spectrum supplied by $\rho_{\vec{c}}^{\text {restr }}(h)$ can be sharpened using wavelet leaders. This explains why one prefers the definition using wavelet leaders (see however Proposition 3.7 below, which shows that some quantities derived from these notions actually coincide).

\section{Robustness criteria}

The wavelet leaders profile of a signal is defined through its wavelet coefficients. The independence from the sufficiently smooth wavelet basis which is chosen is a natural requirement. In practice, one often uses a stronger requirement but easier to handle which implies that the condition considered has some additional stability. This notion was introduced by Meyer in [33] (Chapter 8.9) as follows.

Definition 2.1. If $\gamma$ is a positive number and if $\lambda=\lambda(j, k), \lambda^{\prime}=\lambda\left(j^{\prime}, k^{\prime}\right)$ are two dyadic intervals, let

$$
\omega_{\gamma}\left(\lambda, \lambda^{\prime}\right)=\frac{2^{-\left(\frac{d}{2}+\gamma\right)\left|j-j^{\prime}\right|}}{\left(1+\left(j^{\prime}-j\right)^{2}\right)\left(1+2^{\inf \left\{j, j^{\prime}\right\}} \operatorname{dist}\left(\lambda, \lambda^{\prime}\right)\right)^{\gamma+d}},
$$

where $\operatorname{dist}\left(\lambda, \lambda^{\prime}\right)=\left|k 2^{-j}-k^{\prime} 2^{-j^{\prime}}\right|$. An infinite matrix $A=\left(A\left(\lambda, \lambda^{\prime}\right)\right)_{\left(\lambda, \lambda^{\prime}\right) \in \Lambda \times \Lambda}$ belongs to $\mathcal{A}^{\gamma}$ if there exists $C \geq 0$ such that

$$
\left|A\left(\lambda, \lambda^{\prime}\right)\right| \leq C \omega_{\gamma}\left(\lambda, \lambda^{\prime}\right)
$$

for every $\lambda, \lambda^{\prime} \in \Lambda$. We denote by $\|A\|_{\gamma}$ the infimum of all possible such constants $C$. A matrix is almost diagonal if it belongs to $\mathcal{A}^{\gamma}$ for every $\gamma>0$. Moreover, we say that a matrix is quasidiagonal if it is almost diagonal, invertible on $l^{2}$, and if its inverse is also almost diagonal.

Matrices of operators which map a smooth orthonormal wavelet basis in the Schwartz class into another orthonormal wavelet basis in the Schwartz class are quasidiagonal, see Chapter 8.9 and 10.5 of [33]. Therefore, in order to check that a condition defined on wavelet coefficients is independent of the chosen wavelet basis (in the Schwartz class), one can check the stronger property that it is invariant under the action of quasidiagonal matrices.

Definition 2.2. Let $\mathcal{C}$ be a collection of coefficients indexed by dyadic intervals. A property $\mathcal{P}$ is robust if the following condition holds: If $\mathcal{P}(\mathcal{C})$ holds, then for any quasidiagonal operator $\mathbf{M}, \mathcal{P}(\mathbf{M C})$ holds.

A vector space (or, more generally a subset of a vector space) is robust if it is invariant under the action of quasidiagonal operators.

With a slight abuse of language, we will say that the definition of a quantity $F_{\vec{c}}$ which is a function of a sequence of coefficients $\vec{c}$ is robust if for any quasidiagonal operator $\mathbf{M}, F_{\mathbf{M} \vec{c}}=F_{\vec{c}}$. 
Proposition 2.3. The definition of the wavelet leaders density of a function is not robust.

Proof. We consider again the Cantor set of ratio $\frac{1}{4}$. We define the subset $\Gamma$ of $\Lambda \times \Lambda$ by

$$
\begin{aligned}
\Gamma:=\left\{\left(\lambda, \lambda^{\prime}\right): \exists n \in \mathbb{N} \text { such that } \lambda^{\prime} \subseteq C_{n}\left(\frac{1}{4}\right),\right. \\
{\left.\left[\left(k^{\prime}+3\right) 2^{-j^{\prime}},\left(k^{\prime}+4\right) 2^{-j^{\prime}}\right) \subseteq C_{n}\left(\frac{1}{4}\right) \text { and } j=j^{\prime}+1, k=2 k^{\prime}+3\right\} . }
\end{aligned}
$$

Let us fix $\beta>\alpha>0$ and let us define the infinite matrix $A$ indexed by dyadic intervals by setting

$$
A\left(\lambda, \lambda^{\prime}\right):= \begin{cases}1 & \text { if } \lambda=\lambda^{\prime} \\ 2^{-\beta j} 2^{\alpha j^{\prime}} & \text { if }\left(\lambda, \lambda^{\prime}\right) \in \Gamma \\ 0 & \text { otherwise. }\end{cases}
$$

The matrix $A$ is of the form $I d+R$. Remark that if $\left(\lambda, \lambda^{\prime}\right) \in \Gamma$, then $\left(\lambda^{\prime}, \lambda^{\prime \prime}\right) \notin \Gamma$ for any dyadic interval $\lambda^{\prime \prime}$ and it follows that $R^{2}=0$. This implies that $A$ is invertible, with inverse $I d-R$,

Clearly, the matrices $A$ and $A^{-1}$ belong to $\mathcal{A}^{\gamma}$ for every $\gamma>0$. Let us fix $\delta>\beta$ and let us define the sequence $\vec{c}$ as follows: for every scale $j$ and every dyadic interval $\lambda \in \Lambda_{j}$, we set

$$
c_{\lambda}:= \begin{cases}2^{-\alpha j} & \text { if there exists an } n \in \mathbb{N} \text { such that } \lambda \subseteq C_{n}\left(\frac{1}{4}\right), \\ 0 & \text { if there exists } \lambda^{\prime} \text { such that }\left(\lambda, \lambda^{\prime}\right) \in \Gamma \\ 2^{-\delta j} & \text { otherwise. }\end{cases}
$$

It is straightforward to see that $\rho_{\vec{c}}(\beta)=-\infty$. Let us now consider the image $\vec{x}$ of $\vec{c}$ by the matrix $A$, that is to say

$$
x_{\lambda}=\sum_{\lambda^{\prime}} A\left(\lambda, \lambda^{\prime}\right) c_{\lambda^{\prime}}
$$

Then, if $\lambda \in \Lambda_{j}$, we have

$$
x_{\lambda}:= \begin{cases}2^{-\alpha j} & \text { if there is } n \in \mathbb{N} \text { such that } \lambda \subseteq C_{n}\left(\frac{1}{4}\right), \\ 2^{-\beta j} & \text { if there exists } \lambda^{\prime} \text { such that }\left(\lambda, \lambda^{\prime}\right) \in \Gamma \\ 2^{-\delta j} & \text { otherwise; }\end{cases}
$$

hence $\rho_{\vec{x}}(\beta)=\frac{1}{2}$.

Remark 2.4. It follows from the proof of Proposition 2.3 that the action of a quasidiagonal operator can lift the value of $\rho$ at $\beta$ from $-\infty$ to $1 / 2$, which suffices to prove the result. Note however that the value $-\infty$ does not play a particular role here: Let $\tau<1 / 2$; we can add to the wavelet sequence considered a sequence of $\left\lfloor 2^{\tau j}\right\rfloor$ coefficients located among the vanishing positions, and taking value $2^{-\beta j}$. The initial value of $\rho$ at $\beta$ now is $\tau$; the action of the quasidiagonal operator defined above leaves this new sequence of coefficients unchanged, so that the final value of $\rho$ at $\beta$ is now shifted from $\tau$ to $1 / 2$. 
This counter-example motivates the introduction, in the next section, of another notion based on the wavelet leaders of a signal which will be proved to be robust.

\section{Wavelet leaders profiles}

A theoretical drawback when working with the wavelet leaders density is that it is not a robust quantity. Consequently, it may lead to quantities that are not intrinsic, and therefore not reliable for classification purposes. On the computational side, another drawback comes from the double limit in Definition 1.1. In practice, when dealing with real-life data which are known down to a given scale, one can never really "pass to the limit" several times consecutively, and one must therefore make simultaneously $\varepsilon$ become small and $j$ large, and therefore introduce some dependency between $j$ and $\varepsilon$. However, on the mathematical side, it is easy to check that, as soon as such a dependency between $j$ and $\varepsilon$ is introduced in Definition 1.1 , the value of the corresponding limit can change radically. In other words, this definition is numerically extremely unstable and, in practice, definitions that are based on a single limit are the only ones that can be used. Therefore, we will define another quantity based on the wavelet leaders density which will turn out to be robust and which still yields an upper bound for the multifractal spectrum.

Definition 3.1. The increasing wavelet leaders profile of a sequence $\vec{c} \in C^{0}(\mathbb{T})$ is defined for every $\alpha \in[0,+\infty]$ by

$$
\theta_{\vec{c}}^{+}(\alpha):=\lim _{\varepsilon \rightarrow 0^{+}} \limsup _{j \rightarrow+\infty} \frac{\log \#\left\{\lambda \in \Lambda_{j}: d_{\lambda} \geq 2^{-(\alpha+\varepsilon) j}\right\}}{\log 2^{j}},
$$

where we use the convention that $2^{-\infty}=0$. Similarly, the decreasing wavelet leaders profile of $\vec{c}$ is defined for every $\alpha \geq 0$ by

$$
\theta_{\vec{c}}^{-}(\alpha):=\lim _{\varepsilon \rightarrow 0^{+}} \limsup _{j \rightarrow+\infty} \frac{\log \#\left\{\lambda \in \Lambda_{j}: d_{\lambda} \leq 2^{-(\alpha-\varepsilon) j}\right\}}{\log 2^{j}}
$$

and for $\alpha=+\infty$ by

$$
\theta_{\vec{c}}^{-}(+\infty):=\lim _{A \rightarrow+\infty} \liminf _{j \rightarrow+\infty} \frac{\log \#\left\{\lambda \in \Lambda_{j}: d_{\lambda} \leq 2^{-A j}\right\}}{\log 2^{j}} .
$$

Remark 3.2. The limit over $\varepsilon$ which appears in the definition of the wavelet leaders profile is required in order to derive some mathematical properties that will be useful in the sequel; however, it is not taken into account in applications, and the definition therefore boils down to a single limit, as required, see [16].

As done for the wavelet leaders density, if the sequence $\vec{c}$ is clearly fixed, we will write $\theta^{+}$and $\theta^{-}$instead of $\theta_{\vec{c}}^{+}$and $\theta_{\vec{c}}^{-}$respectively. The next result gives the properties of the increasing and decreasing wavelet leaders profiles of a sequence $\vec{c} \in C^{0}(\mathbb{T})$. 
Proposition 3.3. Let $\vec{c} \in C^{0}(\mathbb{T})$.

1. The increasing wavelet leaders profile $\theta^{+}$of $\vec{c}$ is increasing and right-continuous on $[0,+\infty]$, takes values in $\{-\infty\} \cup[0,1]$, and satisfies $\theta^{+}(+\infty)=1$.

2. The decreasing wavelet leaders profile $\theta^{-}$of $\vec{c}$ is decreasing and left-continuous on $[0,+\infty)$, takes values in $\{-\infty\} \cup[0,1]$, and it satisfies $\theta^{-}(0)=1$.

3. The function

$$
\alpha \in(0,+\infty) \mapsto \frac{\theta^{-}(\alpha)-1}{\alpha}
$$

is decreasing.

Proof. The two first points are immediate. Let us prove the last one. We fix $\alpha, \alpha^{\prime}$ such that $0<\alpha^{\prime}<\alpha$. From the definition of the decreasing wavelet leaders profile of $\vec{c}$, we know that for every $\delta>0$, there is $\varepsilon_{0}>0$ such that for every $0<\varepsilon<\varepsilon_{0}$, there is a sequence $\left(j_{n}\right)_{n \in \mathbb{N}}$ which satisfies

$$
\#\left\{\lambda \in \Lambda_{j_{n}}: d_{\lambda} \leq 2^{-(\alpha-\varepsilon) j_{n}}\right\} \geq 2^{\left(\theta^{-}(\alpha)-\delta\right) j_{n}}, \quad \forall n \in \mathbb{N} .
$$

Then, if $j \geq j_{n}$, we also have

$$
\#\left\{\lambda \in \Lambda_{j}: d_{\lambda} \leq 2^{-(\alpha-\varepsilon) j_{n}}\right\} \geq 2^{j-j_{n}} 2^{\left(\theta^{-}(\alpha)-\delta\right) j_{n}}
$$

since $d_{\lambda} \leq d_{\lambda_{n}}$ if $\lambda \subseteq \lambda_{n}$. For every $n \in \mathbb{N}$, let us set

$$
J_{n}=\left\lfloor\frac{\alpha-\varepsilon}{\alpha^{\prime}-\varepsilon} j_{n}\right\rfloor .
$$

If $n$ is large enough, $J_{n} \geq j_{n}$ and we obtain

$$
\begin{aligned}
\#\left\{\lambda \in \Lambda_{J_{n}}: d_{\lambda} \leq 2^{-\left(\alpha^{\prime}-\varepsilon\right) J_{n}}\right\} & \geq \#\left\{\lambda \in \Lambda_{J_{n}}: d_{\lambda} \leq 2^{-(\alpha-\varepsilon) j_{n}}\right\} \\
& \geq 2^{J_{n}-j_{n}} 2^{\left(\theta^{-}(\alpha)-\delta\right) j_{n}}
\end{aligned}
$$

Consequently,

$$
\begin{aligned}
\limsup _{j \rightarrow \infty} \frac{\log \#\left\{\lambda \in \Lambda_{j}: d_{\lambda}\right.}{\log 2^{j}} & \left.\left.\leq 2^{-\left(\alpha^{\prime}-\varepsilon\right) j}\right\}\right\} \\
& \geq \lim _{n \rightarrow \infty} \frac{\left.\log \#\left\{\lambda \in \Lambda_{J_{n}}: d_{\lambda} \leq 2^{-\left(\alpha^{\prime}-\varepsilon\right) J_{n}}\right\}\right\}}{\log 2^{J_{n}}} \\
& \geq \lim _{n \rightarrow \infty}\left(1+\left(\theta^{-}(\alpha)-\delta-1\right) \frac{j_{n}}{J_{n}}\right) \\
& \geq 1+\left(\theta^{-}(\alpha)-\delta-1\right) \frac{\alpha^{\prime}-\varepsilon}{\alpha-\varepsilon}
\end{aligned}
$$

and it follows that

$$
\theta^{-}\left(\alpha^{\prime}\right) \geq 1+\left(\theta^{-}(\alpha)-\delta-1\right) \frac{\alpha^{\prime}}{\alpha}
$$


Since $\delta>0$ is arbitrary, we get that

$$
\frac{\theta^{-}\left(\alpha^{\prime}\right)-1}{\alpha^{\prime}} \geq \frac{\theta^{-}(\alpha)-1}{\alpha}
$$

hence the conclusion.

Additionally, if there exist $\alpha_{0}>0$ and $C_{0}>0$ (resp. $\alpha_{1}>0$ and $\left.C_{1} \geq 0\right)$ such that

$$
\left|c_{j, k}\right| \leq C_{0} 2^{-\alpha_{0} j} \quad \text { (resp. } \quad d_{j, k} \geq C_{1} 2^{-\alpha_{1} j} \text { ) }
$$

for every $j \in \mathbb{N}_{0}, k \in\left\{0, \ldots, 2^{j}-1\right\}$, then $\theta^{+}$is identically equal to $-\infty$ on $\left(-\infty, \alpha_{0}\right)$ (resp. $\theta^{-}$is identically equal to $-\infty$ on $\left(\alpha_{1},+\infty\right)$ ). Moreover, the increasing and decreasing wavelet profiles of a sequence of wavelet coefficients still yield an upper bound for the spectrum of the corresponding function, as stated in the next result.

Proposition 3.4. Let $f$ be a uniformly Hölder function, and let $\theta^{+}, \theta^{-}$denote the increasing and decreasing wavelet profiles of its sequence of wavelet coefficients in a given wavelet basis. The multifractal spectrum of $f$ satisfies

$$
d_{f}(h) \leq \min \left\{\theta^{+}(h), \theta^{-}(h)\right\} \quad \forall h \in[0,+\infty] .
$$

Proof. It is clear that $\theta^{+}(h) \geq \rho(h)$ and $\theta^{-}(h) \geq \rho(h)$ for every $h$. The result follows then directly from Theorem 1.5.

The following lemma shows the link between the wavelet leaders density of a function and its wavelet leaders profiles.

\section{Lemma 3.5.}

1. If $\vec{c} \in C^{0}(\mathbb{T})$, then,

$$
\theta^{+}(\alpha)=\sup _{\alpha^{\prime} \leq \alpha} \rho\left(\alpha^{\prime}\right), \quad \forall \alpha \in[0,+\infty]
$$

2. Assume that $\vec{c} \in C^{0}(\mathbb{T})$ is a sequence for which there are $\alpha_{1}>0$ and $C_{1} \geq 0$ such that $d_{j, k} \geq C_{1} 2^{-\alpha_{1} j}$ for every $j \in \mathbb{N}_{0}, k \in\left\{0, \ldots, 2^{j}-1\right\}$. Then,

$$
\theta^{-}(\alpha)=\sup _{\alpha^{\prime} \geq \alpha} \rho\left(\alpha^{\prime}\right), \quad \forall \alpha \in[0,+\infty] .
$$

Proof. 1. Let $\alpha_{0}=\inf \left\{\alpha \geq 0: \theta^{+}(\alpha) \geq 0\right\}$. The result is clear if $\alpha<\alpha_{0}$. So, let us assume that $\alpha \geq \alpha_{0}$. Of course, we have $\theta^{+}(\alpha) \geq \rho(\alpha)$. Since $\theta^{+}$is increasing, we get that

$$
\theta^{+}(\alpha) \geq \sup _{\alpha^{\prime} \leq \alpha} \rho\left(\alpha^{\prime}\right)
$$

For the other inequality, let us fix $\varepsilon>0$. By definition of $\rho$, for every $\alpha^{\prime} \leq \alpha+\varepsilon$, there exist $r\left(\alpha^{\prime}\right)>0$ and $J\left(\alpha^{\prime}\right) \in \mathbb{N}$ such that

$$
\#\left\{\lambda \in \Lambda_{j}: d_{\lambda} \geq 2^{-\left(\alpha^{\prime}+r\left(\alpha^{\prime}\right)\right) j}\right\}-\#\left\{\lambda \in \Lambda_{j}: d_{\lambda} \geq 2^{-\left(\alpha^{\prime}-r\left(\alpha^{\prime}\right)\right) j}\right\} \leq 2^{\left(\rho\left(\alpha^{\prime}\right)+\varepsilon\right) j}
$$


for every $j \geq J\left(\alpha^{\prime}\right)$. From the covering of the compact $\left[\alpha_{0}, \alpha+\varepsilon\right]$ by the open sets $\left(\alpha^{\prime}-r\left(\alpha^{\prime}\right), \alpha^{\prime}+r\left(\alpha^{\prime}\right)\right)$, we extract a finite subcovering $\left\{\left(\alpha_{i}^{\prime}-r\left(\alpha_{i}^{\prime}\right), \alpha_{i}^{\prime}+r\left(\alpha_{i}^{\prime}\right)\right)\right.$ : $i \in\{1, \ldots, n\}\}$. Fix $J \geq \max _{1 \leq i \leq n} J\left(\alpha_{i}^{\prime}\right)$. For every $j \geq J$, we have

$$
\begin{aligned}
& \#\left\{\lambda \in \Lambda_{j}: d_{\lambda} \geq 2^{-(\alpha+\varepsilon) j}\right\} \\
\leq & \sum_{i=1}^{n} \#\left\{\lambda \in \Lambda_{j}: d_{\lambda} \geq 2^{-\left(\alpha_{i}^{\prime}+r\left(\alpha_{i}^{\prime}\right)\right) j}\right\}-\#\left\{\lambda \in \Lambda_{j}: d_{\lambda} \geq 2^{-\left(\alpha_{i}^{\prime}-r\left(\alpha_{i}^{\prime}\right)\right) j}\right\} \\
\leq & \sum_{i=1}^{n} 2^{\left(\rho\left(\alpha_{i}^{\prime}\right)+\varepsilon\right) j} \leq n 2^{\left(\sup _{\alpha^{\prime} \leq \alpha} \rho\left(\alpha^{\prime}\right)+\varepsilon\right) j} .
\end{aligned}
$$

It follows directly that $\theta^{+}(\alpha) \leq \sup _{\alpha^{\prime} \leq \alpha} \rho\left(\alpha^{\prime}\right)$. We still have to consider the case where $\alpha=+\infty$. It follows from the fact that $\theta^{+}(+\infty)=1$ and $\sup _{\alpha \geq 0} \rho(\alpha)=1$.

2 . The proof of the second part is very similar.

Remark 3.6. Consequently, in practice, in order to estimate the multifractal spectrum of a function using the wavelet profile of its sequence of wavelet coefficients in a given wavelet basis, one can proceed as follows: Denote by $\alpha_{s}$ the smallest positive number such that $\theta^{+}\left(\alpha_{s}\right)=1$. Then, the spectrum is estimated by $\theta^{+}(\alpha)$ if $\alpha<\alpha_{s}$ and by $\theta^{-}(\alpha)$ if $\alpha \geq \alpha_{s}$.

The next proposition shows that one could define equivalently the increasing and decreasing wavelet leaders profiles of a sequence using the restricted wavelet leaders $e_{\lambda}$ instead of the wavelet leaders $d_{\lambda}$.

Proposition 3.7. If $\vec{c} \in C^{0}(\mathbb{T})$, then for every $\alpha \in[0,+\infty]$,

$$
\theta^{+}(\alpha)=\lim _{\varepsilon \rightarrow 0^{+}} \limsup _{j \rightarrow+\infty} \frac{\log \#\left\{\lambda \in \Lambda_{j}: e_{\lambda} \geq 2^{-(\alpha+\varepsilon) j}\right\}}{\log 2^{j}} .
$$

Moreover, for every $\alpha \in[0,+\infty)$,

$$
\theta^{-}(\alpha)=\lim _{\varepsilon \rightarrow 0^{+}} \limsup _{j \rightarrow+\infty} \frac{\log \#\left\{\lambda \in \Lambda_{j}: e_{\lambda} \leq 2^{-(\alpha-\varepsilon) j}\right\}}{\log 2^{j}},
$$

and for $\alpha=+\infty$,

$$
\theta^{-}(+\infty)=\lim _{A \rightarrow+\infty} \liminf _{j \rightarrow+\infty} \frac{\log \#\left\{\lambda \in \Lambda_{j}: e_{\lambda} \leq 2^{-A j}\right\}}{\log 2^{j}} .
$$

Proof. 1. Define $\theta^{+, \text {restr }}(\alpha)$ as the right hand side of (3.1). Then it is clear that $\theta^{+, \text {restr }}(\alpha) \leq \theta^{+}(\alpha)$ for every $\alpha \in[0,+\infty]$ since $e_{\lambda} \leq d_{\lambda}$ for every dyadic interval $\lambda$. For the other inequality, let us fix $\alpha \in[0,+\infty)$ and $\delta>0$. By definition, there exist $J \geq 0$ and $\varepsilon>0$ such that

$$
\#\left\{\lambda \in \Lambda_{j}: e_{\lambda} \geq 2^{-(\alpha+\varepsilon) j}\right\} \leq 2^{\left(\theta^{+, \text {restr }}(\alpha)+\delta\right) j} \quad \forall j \geq J .
$$


Let us fix $j \geq J$. As before, for all $\lambda \in \Lambda_{j}$, we denote $N(\lambda)$ the set of the 3 "neighbors" of $\lambda$ in $\Lambda_{j}$. Then we have

$$
d_{\lambda}=\max \left\{e_{\mu}: \mu \in N(\lambda)\right\}
$$

and it follows that, for $j$ large enough,

$$
\begin{aligned}
\#\left\{\lambda \in \Lambda_{j}: d_{\lambda} \geq 2^{-(\alpha+\varepsilon) j}\right\} & \leq 3 \cdot \#\left\{\lambda \in \Lambda_{j}: e_{\lambda} \geq 2^{-(\alpha+\varepsilon) j}\right\} \\
& \leq 3 \cdot 2^{\left(\theta^{+, \text {restr }}(\alpha)+\delta\right) j} .
\end{aligned}
$$

Thus $\theta^{+}(\alpha) \leq \theta^{+, \text {restr }}(\alpha)+\delta$ and since $\delta>0$ is arbitrary, we get the conclusion if $\alpha$ is finite. The result is also true for $\alpha=+\infty$ because at this point, these two functions take the value one.

2. The proof of the second point is similar.

Consequently, we will often work with restricted wavelet leaders instead of wavelet leaders. This will be the case in the next section. Both functions $\theta^{+}$and $\theta^{+, \text {restr }}$ will be denoted by $\theta^{+}$. We use similar notations for the decreasing profile. Let us mention that the " $3 \lambda$ " in the definition of the wavelet leaders comes from Proposition 1.2, which is no longer valid in the case of restricted wavelet leaders.

Let us end this section by mentioning that the increasing leaders profile $\theta^{+}$ (defined using wavelet leaders) of a sequence has been theoretically compared with its wavelet profile $\nu$ (defined using wavelet coefficients) in [16]: the inequality $\theta^{+} \leq \nu$ is of course always satisfied, and a sufficient and necessary condition to have the strict inequality has been given.

\section{Robustness of the wavelet leaders profile}

Let $\vec{c} \in \Omega$ and let $A$ be a quasidiagonal matrix. We define $\vec{x}$ by setting

$$
x_{\lambda}=\sum_{\lambda^{\prime} \in \Lambda} A\left(\lambda, \lambda^{\prime}\right) c_{\lambda^{\prime}}
$$

for every dyadic interval $\lambda$. The aim of this section is to prove that $\theta_{\vec{c}}^{+}(\alpha)=\theta_{\vec{x}}^{+}(\alpha)$ and $\theta_{\vec{c}}^{-}(\alpha)=\theta_{\vec{x}}^{-}(\alpha)$ for every $\alpha \in[0,+\infty]$. Let us first recall a lemma from [33].

Lemma 4.1. Let $\gamma>|\alpha|$ and $A \in \mathcal{A}^{\gamma}$. There exists a constant $\widetilde{C}$ such that

$$
\left|c_{j, k}\right| \leq C 2^{-\alpha j} \quad \forall j, k \Rightarrow\left|x_{j, k}\right| \leq \widetilde{C}\|A\|_{\gamma} C 2^{-\alpha j} \quad \forall j, k .
$$

This lemma expresses the fact that operators whose matrix in a wavelet basis belongs to $\mathcal{A}^{\gamma}$ are continuous on $C^{\alpha}(\mathbb{T})$ if $|\alpha|<\gamma$. It is a straightforward consequence of the proof of Schur's lemma (Lemma 4 in Chapter 8, [33]). 
Definition 4.2. Let $\varepsilon>0$ and let $\lambda$ be a dyadic interval. The $\varepsilon$-neighborhood of $\lambda$, denoted by $N^{\varepsilon}(\lambda)$, is the set of dyadic intervals $\lambda^{\prime}$ such that

$$
\left\{\begin{array}{l}
\left|j-j^{\prime}\right| \leq \varepsilon j, \\
\left|\frac{k}{2^{j}}-\frac{k^{\prime}}{2^{j^{\prime}}}\right| \leq 2^{2 \varepsilon j} 2^{-j} .
\end{array}\right.
$$

Remark 4.3. Note that if $\lambda^{\prime}$ does not belong to $N^{\varepsilon}(\lambda)$ and if $\gamma \geq \varepsilon^{-2}$, a computation leads to

$$
\omega_{2 \gamma}\left(\lambda, \lambda^{\prime}\right) \leq \omega_{\gamma}\left(\lambda, \lambda^{\prime}\right) 2^{-j / \varepsilon}
$$

Proposition 4.4. Let $\vec{c} \in C^{r}(\mathbb{T})$ for some $r>0$. The definition of the increasing wavelet leaders profile of $\vec{c}$ is robust.

Proof. Let $A$ be a quasidiagonal matrix and consider

$$
x_{\lambda}=\sum_{\lambda^{\prime} \in \Lambda} A\left(\lambda, \lambda^{\prime}\right) c_{\lambda^{\prime}}
$$

for every dyadic interval $\lambda$. Let us first show that $\theta_{\vec{x}}^{+}(\alpha) \leq \theta_{\vec{c}}^{+}(\alpha)$ for every $\alpha \in$ $[0,+\infty]$. Let us denote $\alpha_{\min }=\inf \left\{\alpha: \theta_{\vec{c}}^{+}(\alpha) \geq 0\right\}$. Since $\vec{c} \in C^{r}(\mathbb{T})$, we know that $\alpha_{\min }>0$.

1. Assume that $\alpha<\alpha_{\min }$.

If $\varepsilon>0$ is such that $\alpha+\varepsilon<\alpha_{\text {min }}$, there is $C_{1}>0$ with

$$
\left|c_{j, k}\right| \leq C_{1} 2^{-(\alpha+\varepsilon) j} \quad \forall j \in \mathbb{N}_{0}, k \in\left\{0, \ldots, 2^{j}-1\right\} .
$$

Therefore, Lemma 4.1 implies that $\left|x_{j, k}\right| \leq \widetilde{C}\|A\|_{\gamma} C_{1} 2^{-(\alpha+\varepsilon) j}$ for $\gamma>\alpha+\varepsilon$ and for every $j \in \mathbb{N}_{0}$ and $k \in\left\{0, \ldots, 2^{j}-1\right\}$. We then directly obtain that $\theta_{\vec{x}}^{+}(\alpha)=-\infty$ for every $\alpha<\alpha_{\min }$.

\section{Assume that $\alpha \geq \alpha_{\min }>0$.}

Let us fix $\delta>0$. We will prove that there exist $J \in \mathbb{N}$ and $\varepsilon>0$ such that

$$
\#\left\{\lambda \in \Lambda_{l}: \sup _{\lambda^{\prime} \subseteq \lambda}\left|x_{\lambda^{\prime}}\right| \geq 2^{-(\alpha+\varepsilon) l}\right\} \leq 2^{\left(\theta_{\vec{c}}^{+}(\alpha)+8 \delta\right) l}
$$

for all $l \geq J$. Since $\delta>0$ is arbitrary, we will get that $\theta_{\vec{x}}^{+}(\alpha) \leq \theta_{\vec{c}}^{+}(\alpha)$.

Using the right continuity of $\theta_{\vec{c}}^{+}$, we choose $\varepsilon>0$ such that $\varepsilon<\delta, \alpha-\alpha_{\min }<\varepsilon^{-1}$ and $\theta_{\vec{c}}^{+}\left(\frac{\alpha}{1-\varepsilon}\right) \leq \theta_{\vec{c}}^{+}(\alpha)+\delta$. The definition of $\theta_{\vec{c}}^{+}$gives $\varepsilon_{0}>0$ and $J \in \mathbb{N}_{0}$ such that

$$
\#\left\{\lambda \in \Lambda_{j}: \sup _{\lambda^{\prime} \subseteq \lambda}\left|c_{\lambda^{\prime}}\right| \geq 2^{-\left(\frac{\alpha}{1-\varepsilon}+2 \varepsilon_{0}\right) j}\right\} \leq 2^{\left(\theta_{\vec{c}}^{+}\left(\frac{\alpha}{1-\varepsilon}\right)+\delta\right) j}
$$


for every $j \geq J$. Of course, we can also assume that $\varepsilon_{0}$ is small enough so that $\alpha+\varepsilon_{0}(1-\varepsilon)-\alpha_{\min }<\varepsilon^{-1}$.

For every $l \in \mathbb{N}_{0}$, we define

$$
\Lambda_{1}=\Lambda_{1}(l, \varepsilon)=\bigcup_{(1-\varepsilon) l \leq j \leq(1+\varepsilon) l}\left\{\lambda \in \Lambda_{j}: \sup _{\lambda^{\prime} \subseteq \lambda}\left|c_{\lambda^{\prime}}\right| \geq \frac{1}{4 \widetilde{C}\|A\|_{\alpha}} 2^{-\left(\frac{\alpha}{1-\varepsilon}+\varepsilon_{0}\right) j}\right\}
$$

and

$$
\Lambda_{2}=\Lambda_{2}(l, \varepsilon)=\left\{\lambda \in \Lambda_{l}: \exists \lambda_{1} \in \Lambda_{1} \text { with }\left|\frac{k_{1}}{2^{j_{1}}}-\frac{k}{2^{l}}\right| \leq 2^{-l+1} 2^{2 \varepsilon l}\right\} .
$$

a) Let us show that if $\lambda_{0} \notin \Lambda_{2}(l, \varepsilon)$ is of size $2^{-l}$, then

$$
\sup _{\lambda \subseteq \lambda_{0}}\left|x_{\lambda}\right|<2^{-\left(\alpha+\varepsilon_{0}(1-\varepsilon)\right) l} .
$$

It suffices to show that if $\lambda \subseteq \lambda_{0}$, then

$$
\left|x_{\lambda}\right|=\left|\sum_{\lambda^{\prime}} A\left(\lambda, \lambda^{\prime}\right) c_{\lambda^{\prime}}\right|<\frac{1}{2} 2^{-\left(\alpha+\varepsilon_{0}(1-\varepsilon)\right) l} .
$$

So, let us fix $\lambda=\lambda(j, k) \subseteq \lambda_{0}=\lambda\left(l, k_{0}\right)$. Remark that in particular, we have $j \geq l$. We set

$$
y_{\lambda}=\sum_{\lambda^{\prime} \nsubseteq \lambda_{1} \forall \lambda_{1} \in \Lambda_{1} \text { and }} A\left(\lambda, \lambda^{\prime}\right) c_{\lambda^{\prime}}
$$

and

$$
z_{\lambda}=\sum_{\lambda^{\prime} \subseteq \lambda_{1} \text { with } \lambda_{1} \in \Lambda_{1} \text { or } j^{\prime}<(1-\varepsilon) l} A\left(\lambda, \lambda^{\prime}\right) c_{\lambda^{\prime}}
$$

so that we have $\left|x_{\lambda}\right| \leq\left|y_{\lambda}\right|+\left|z_{\lambda}\right|$.

- If $\lambda^{\prime}$ is such that $\lambda^{\prime} \nsubseteq \lambda_{1}$ for any $\lambda_{1} \in \Lambda_{1}$ and if $j^{\prime} \geq(1-\varepsilon) l$, then by definition of $\Lambda_{1}(l, \varepsilon)$, we have

$$
\left|c_{\lambda^{\prime}}\right|<\frac{1}{4 \widetilde{C}\|A\|_{\alpha}} 2^{-\left(\frac{\alpha}{1-\varepsilon}+\varepsilon_{0}\right) l(1-\varepsilon)}=\frac{1}{4 \widetilde{C}\|A\|_{\alpha}} 2^{-\left(\alpha+\varepsilon_{0}(1-\varepsilon)\right) l} .
$$

Using Lemma 4.1 with $\alpha>0$, we get that

$$
\left|y_{\lambda}\right| \leq \frac{1}{4 \widetilde{C}\|A\|_{\alpha}} 2^{-\left(\alpha+\varepsilon_{0}(1-\varepsilon)\right) l} \widetilde{C}\|A\|_{\alpha} 2^{-0 j}=\frac{1}{4} 2^{-\left(\alpha+\varepsilon_{0}(1-\varepsilon)\right) l} .
$$

- If $\lambda^{\prime}$ is such that there is $\lambda_{1} \in \Lambda_{1}(l, \varepsilon)$ with $\lambda^{\prime} \subseteq \lambda_{1}$ or if $j^{\prime}<(1-\varepsilon) l$, let us show that $\lambda^{\prime} \notin N^{\varepsilon}(\lambda)$. First, if $j^{\prime}<(1-\varepsilon) l$, then $j^{\prime}<(1-\varepsilon) j$ since $j \geq l$ and 
it follows that $\lambda^{\prime} \notin N^{\varepsilon}(\lambda)$. So we can assume that $\lambda^{\prime} \subseteq \lambda_{1}$ with $\lambda_{1} \in \Lambda_{1}$. Since $\lambda_{1} \in \Lambda_{1}$ and $\lambda_{0} \notin \Lambda_{2}$, we know that

$$
\left|\frac{k_{0}}{2^{l}}-\frac{k_{1}}{2^{j_{1}}}\right|>2^{-l+1} 2^{2 \varepsilon l} .
$$

Let us first assume that

$$
\frac{k_{0}}{2^{l}}-\frac{k_{1}}{2^{j_{1}}}>2^{-l+1} 2^{2 \varepsilon l} .
$$

From the inclusions $\lambda \subset \lambda_{0}$ and $\lambda^{\prime} \subset \lambda_{1}$, we have

$$
\frac{k_{0}}{2^{l}}-\frac{k_{1}}{2^{j_{1}}} \leq \frac{k}{2^{j}}-\frac{k^{\prime}}{2^{j^{\prime}}}-\frac{1}{2^{j^{\prime}}}+\frac{1}{2^{j_{1}}} \leq \frac{k}{2^{j}}-\frac{k^{\prime}}{2^{j^{\prime}}}+\frac{1}{2^{j_{1}}}
$$

and consequently, from the previous relation,

$$
\frac{k}{2^{j}}-\frac{k^{\prime}}{2^{j^{\prime}}}>2^{-l+1} 2^{2 \varepsilon l}-\frac{1}{2^{j_{1}}} .
$$

Moreover, since $\lambda_{1} \in \Lambda_{1}(l, \varepsilon)$, we have $j_{1} \geq(1-\varepsilon) l$ and it follows that

$$
\begin{aligned}
\frac{k}{2^{j}}-\frac{k^{\prime}}{2^{j^{\prime}}}>2^{-l+1} 2^{2 \varepsilon l}-2^{-((1-\varepsilon) l)} & =2^{(2 \varepsilon-1) l}+2^{(2 \varepsilon-1) l}-2^{-((1-\varepsilon) l)} \\
& \geq 2^{(2 \varepsilon-1) l} \geq 2^{(2 \varepsilon-1) j}
\end{aligned}
$$

where the last inequality comes from the fact that $j \geq l$ and $2 \varepsilon-1<0$.

The second case is quite similar. Assume that

$$
\frac{k_{1}}{2^{j_{1}}}-\frac{k_{0}}{2^{l}}>2^{-l+1} 2^{2 \varepsilon l} .
$$

Using inclusions between dyadic intervals, we have

$$
\frac{k_{1}}{2^{j_{1}}}-\frac{k_{0}}{2^{l}} \leq \frac{k^{\prime}}{2^{j^{\prime}}}-\frac{k}{2^{j}}-\frac{1}{2^{j}}+\frac{1}{2^{l}} \leq \frac{k^{\prime}}{2^{j^{\prime}}}-\frac{k}{2^{j}}+\frac{1}{2^{l}}
$$

and it follows that

$$
\frac{k^{\prime}}{2^{j^{\prime}}}-\frac{k}{2^{j}}>2^{-l+1} 2^{2 \varepsilon l}-\frac{1}{2^{l}} \geq 2^{(2 \varepsilon-1) l} \geq 2^{(2 \varepsilon-1) j} .
$$

So, we have proved that $\lambda^{\prime} \notin N^{\varepsilon}(\lambda)$.

Consequently, using Remark 4.3, we get

$$
\begin{aligned}
\left|z_{\lambda}\right| & \leq \sum_{\lambda^{\prime}: \lambda^{\prime} \notin N^{\varepsilon}(\lambda)}\left|A\left(\lambda, \lambda^{\prime}\right)\right|\left|c_{\lambda^{\prime}}\right| \\
& \leq \sum_{\lambda^{\prime}: \lambda^{\prime} \notin N^{\varepsilon}(\lambda)}\|A\|_{2 \varepsilon^{-2}} \omega_{2 \varepsilon^{-2}}\left(\lambda, \lambda^{\prime}\right)\left|c_{\lambda^{\prime}}\right| \\
& \leq\|A\|_{2 \varepsilon^{-2}} \sum_{\lambda^{\prime}: \lambda^{\prime} \notin N^{\varepsilon}(\lambda)} \omega_{\varepsilon^{-2}}\left(\lambda, \lambda^{\prime}\right) 2^{-j \varepsilon^{-1}}\left|c_{\lambda^{\prime}}\right| \\
& \leq\|A\|_{2 \varepsilon^{-2}} 2^{-j \varepsilon^{-1}} C_{1} \sum_{\lambda^{\prime}: \lambda^{\prime} \notin N^{\varepsilon}(\lambda)} \omega_{\varepsilon^{-2}}\left(\lambda, \lambda^{\prime}\right) 2^{-\alpha_{0} j^{\prime}}
\end{aligned}
$$


where $\alpha_{0}<\alpha_{\min }$ is such that $\alpha+\varepsilon_{0}(1-\varepsilon)-\alpha_{0}<\varepsilon^{-1}$ and the constant $C_{1}>0$ is such that $\left|c_{j, k}\right| \leq C_{1} 2^{-\alpha_{0} j}$ for every $j \in \mathbb{N}, k \in\left\{0, \ldots, 2^{j}-1\right\}$. Lemma 4.1 gives

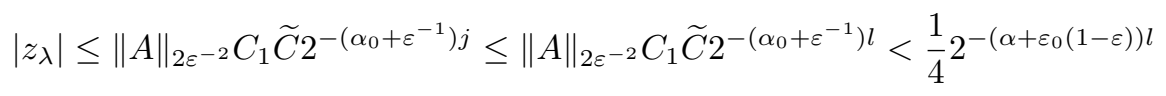

if $l$ is large enough.

Finally, we have obtained

$$
\left|x_{\lambda}\right| \leq\left|y_{\lambda}\right|+\left|z_{\lambda}\right| \leq \frac{1}{2} 2^{-\left(\alpha+\varepsilon_{0}(1-\varepsilon)\right) l}
$$

if $l$ is large enough. It follows that if $\lambda_{0} \notin \Lambda_{2}(l, \varepsilon)$ is of size $2^{-l}$, then $\sup _{\lambda \subseteq \lambda_{0}}\left|x_{\lambda}\right|<$ $2^{-\left(\alpha+\varepsilon_{0}(1-\varepsilon)\right) l}$. So,

$$
\#\left\{\lambda_{0} \in \Lambda_{l}: \sup _{\lambda \subseteq \lambda_{0}}\left|x_{\lambda_{0}}\right| \geq 2^{-\left(\alpha+\varepsilon_{0}(1-\varepsilon)\right) l}\right\} \leq \# \Lambda_{2}(l, \varepsilon) .
$$

b) Estimation of the cardinality of $\Lambda_{2}(l, \varepsilon)$

Remark first that if $\lambda_{1}=\lambda\left(j_{1}, k_{1}\right) \in \Lambda_{1}(l, \varepsilon)$ is fixed, we have

$$
\begin{aligned}
& \#\left\{k \in\left\{0, \ldots, 2^{l}-1\right\}:\left|\frac{k}{2^{l}}-\frac{k_{1}}{2^{j_{1}}}\right| \leq 2^{-l+1} 2^{2 \varepsilon l}\right\} \\
= & \#\left\{k \in\left\{0, \ldots, 2^{l}-1\right\}: k_{1} 2^{l-j_{1}}-2^{1+2 \varepsilon l} \leq k \leq k_{1} 2^{l-j_{1}}+2^{1+2 \varepsilon l}\right\} \\
\leq & k_{1} 2^{l-j_{1}}+2^{1+2 \varepsilon l}-k_{1} 2^{l-j_{1}}+2^{1+2 \varepsilon l}+1=2^{2 \varepsilon l+2}+1 \leq 2^{3 \varepsilon l}
\end{aligned}
$$

if $l$ is large enough. Therefore, we get

$$
\# \Lambda_{2}(l, \varepsilon) \leq \sum_{(1-\varepsilon) l \leq j_{1} \leq(1+\varepsilon) l} \#\left\{\lambda \in \Lambda_{j_{1}}: \sup _{\lambda^{\prime} \subseteq \lambda}\left|c_{\lambda^{\prime}}\right| \geq \frac{1}{4 \widetilde{C}\|A\|_{\alpha}} 2^{-\left(\frac{\alpha}{1-\varepsilon}+\varepsilon_{0}\right) j_{1}}\right\} .
$$

Moreover,

$$
\begin{aligned}
& \#\left\{\lambda \in \Lambda_{j_{1}}: \sup _{\lambda^{\prime} \subseteq \lambda}\left|c_{\lambda^{\prime}}\right| \geq \frac{1}{4 \widetilde{C}\|A\|_{\alpha}} 2^{-\left(\frac{\alpha}{1-\varepsilon}+\varepsilon_{0}\right) j_{1}}\right\} \\
\leq & \#\left\{\lambda \in \Lambda_{j_{1}}: \sup _{\lambda^{\prime} \subseteq \lambda}\left|c_{\lambda^{\prime}}\right| \geq 2^{-\left(\frac{\alpha}{1-\varepsilon}+2 \varepsilon_{0}\right) j_{1}}\right\} \\
\leq & 2^{\left(\theta_{\vec{c}}^{+}\left(\frac{\alpha}{1-\varepsilon}\right)+\delta\right) j_{1}} \leq 2^{\left(\theta_{\vec{c}}^{+}(\alpha)+2 \delta\right) j_{1}}
\end{aligned}
$$


for $j_{1}$ (hence $l$ ) large enough. It follows that

$$
\begin{aligned}
& \# \quad\left\{\lambda_{0} \in \Lambda_{l}: \sup _{\lambda \subseteq \lambda_{0}}\left|x_{\lambda}\right| \geq 2^{-\left(\alpha+\varepsilon_{0}(1-\varepsilon)\right) l}\right\} \\
& \leq \quad \sum_{(1-\varepsilon) l \leq j_{1} \leq(1+\varepsilon) l} 2^{\left(\theta_{\vec{c}}^{+}(\alpha)+2 \delta\right) j_{1}} 2^{3 \varepsilon l} \\
& \leq \quad \sum_{(1-\varepsilon) l \leq j_{1} \leq(1+\varepsilon) l} 2^{\left(\theta_{\vec{c}}^{+}(\alpha)+2 \delta\right)(1+\varepsilon) l} 2^{3 \varepsilon l} \\
& \leq \quad(2 \varepsilon l+1) 2^{\left(\theta_{\vec{c}}^{+}(\alpha)+2 \delta+\varepsilon\left(\theta_{\vec{c}}^{+}(\alpha)+2 \delta+3\right)\right) l} \\
& \leq 2^{\varepsilon l} 2^{\left(\theta_{\vec{c}}^{+}(\alpha)+2 \delta+5 \varepsilon\right) l} \\
& \leq 2^{\left(\theta_{\vec{c}}^{+}(\alpha)+8 \delta\right) l}
\end{aligned}
$$

if $l$ is large enough.

So, we have proved that for every $\alpha \in[0,+\infty], \theta_{\vec{x}}^{+}(\alpha) \leq \theta_{\vec{c}}^{+}(\alpha)$. We have also obtained that $\inf \left\{\alpha: \theta_{\vec{x}}^{+}(\alpha) \geq 0\right\} \geq \alpha_{\min }>0$. Since $A^{-1}$ is also almost diagonal, the same proof shows that $\theta_{\vec{c}}^{+}(\alpha) \leq \theta_{\vec{x}}^{+}(\alpha)$ for every $\alpha \in \mathbb{R}$. The conclusion follows.

In order to prove the robustness of the decreasing wavelet leaders profile, let us introduce a new notation. Let us fix a dyadic interval $\lambda_{0}\left(l, k_{0}\right)$ and $\varepsilon>0$. For $j \in \mathbb{N}_{0}$ and $k \in\left\{0, \ldots, 2^{j}-1\right\}$,

$\lambda(j, k) \in \operatorname{Cond}_{\varepsilon}\left(\lambda_{0}\right) \Longleftrightarrow(1-2 \varepsilon) j>l$ and $2^{(2 \varepsilon-1) j} \leq \frac{k}{2^{j}}-\frac{k_{0}}{2^{l}} \leq 2^{-l}-3 \cdot 2^{(2 \varepsilon-1) j}$.

Lemma 4.5. Let us fix a dyadic interval $\lambda_{0}\left(k_{0}, l\right)$ and let us consider $\varepsilon>0$. If $\lambda(j, k) \in \operatorname{Cond}_{\varepsilon}\left(\lambda_{0}\right)$, then

$$
\lambda^{\prime} \in N^{\varepsilon}(\lambda(j, k)) \Longrightarrow \lambda^{\prime} \subset \lambda_{0}
$$

Proof. First, we have

$$
j^{\prime} \geq(1-\varepsilon) j \geq(1-2 \varepsilon) j>l .
$$

Moreover,

Finally, we have

$$
\frac{k^{\prime}}{2^{j^{\prime}}} \geq \frac{k}{2^{j}}-2^{(2 \varepsilon-1) j} \geq \frac{k_{0}}{2^{l}}
$$

$$
\begin{aligned}
\frac{k^{\prime}+1}{2^{j^{\prime}}} \leq \frac{1}{2^{j^{\prime}}}+\frac{k}{2^{j}}+2^{(2 \varepsilon-1) j} & \leq 2^{-j^{\prime}}+\frac{k_{0}}{2^{l}}+2^{-l}-3 \cdot 2^{(2 \varepsilon-1) j}+2^{(2 \varepsilon-1) j} \\
& \leq \frac{k_{0}+1}{2^{l}}-2^{(2 \varepsilon-1) j} \leq \frac{k_{0}+1}{2^{l}}
\end{aligned}
$$


Proposition 4.6. Let $\vec{c} \in C^{r}(\mathbb{T})$ for some $r>0$. The definition of the decreasing wavelet leaders profile of $\vec{c}$ is robust.

Proof. Let $A$ be a quasidiagonal matrix and

$$
x_{\lambda}=\sum_{\lambda^{\prime} \in \Lambda} A\left(\lambda, \lambda^{\prime}\right) c_{\lambda^{\prime}}
$$

for every dyadic interval $\lambda$. Let us first remark that, as done in the case of the proof of Proposition 4.4, since $\vec{c} \in C^{r}(\mathbb{T})$, there exists $C_{1}>0$ such that $\left|x_{j, k}\right| \leq C_{1} 2^{-r j}$ for every $j \in \mathbb{N}, k \in\left\{0, \ldots, 2^{j}-1\right\}$. In particular, we have $\theta_{\vec{x}}^{-}(\alpha)=\theta_{\vec{c}}^{-}(\alpha)=-\infty$ if $\alpha \leq r$.

Let us show that $\theta_{\vec{x}}^{-}(\alpha) \leq \theta_{\vec{c}}^{-}(\alpha)$ for every $\alpha>r$. Since $A$ is quasidiagonal, we will obtain the other inequality with the same arguments.

Let us fix $\varepsilon \in\left(0, \frac{1}{2}\right)$ small enough so that $\alpha-r<\varepsilon^{-1}$. Since $\alpha<r+\varepsilon^{-1}$, there is $J \in \mathbb{N}$ such that

$$
\left\|A^{-1}\right\|_{2 \varepsilon^{-2}} C_{1} 2^{-\left(r+\varepsilon^{-1}\right) l} \leq\left\|A^{-1}\right\|_{\alpha} 2^{-\alpha l}
$$

for every $l \geq J$. For $\varepsilon_{0}>0$ small enough, we have $\alpha-r+\varepsilon_{0}(1+\varepsilon)<\varepsilon^{-1}$ and for every $l \geq J$, we define

$$
E_{l}=\left\{\lambda_{0} \in \Lambda_{l}: \sup _{\lambda \in \operatorname{Cond}_{\varepsilon}\left(\lambda_{0}\right)}\left|c_{\lambda}\right| \leq 2 \widetilde{C}\left\|A^{-1}\right\|_{\alpha} 2^{-\left(\alpha-\varepsilon_{0}(1+\varepsilon)\right) l}\right\} .
$$

Let us show that if $\lambda_{0} \in \Lambda_{l}$ is such that $\sup _{\lambda^{\prime} \subset \lambda_{0}}\left|x_{\lambda^{\prime}}\right| \leq 2^{-\left(\alpha-\varepsilon_{0}(1+\varepsilon)\right) l}$, then $\lambda_{0} \in E_{l}$. Let us fix $\lambda \in \operatorname{Cond}_{\varepsilon}\left(\lambda_{0}\right)$. We have

$$
\left|c_{\lambda}\right| \leq\left|\sum_{\lambda^{\prime} \in N^{\varepsilon}(\lambda)} A^{-1}\left(\lambda, \lambda^{\prime}\right) x_{\lambda^{\prime}}\right|+\left|\sum_{\lambda^{\prime} \notin N^{\varepsilon}(\lambda)} A^{-1}\left(\lambda, \lambda^{\prime}\right) x_{\lambda^{\prime}}\right| .
$$

As done in Proposition 4.4, using the Remark 4.3, we have

$$
\left|\sum_{\lambda^{\prime} \notin N^{\varepsilon}(\lambda)} A^{-1}\left(\lambda, \lambda^{\prime}\right) x_{\lambda^{\prime}}\right| \leq\left\|A^{-1}\right\|_{2 \varepsilon^{-2}} C_{1} \widetilde{C} 2^{-\left(r+\varepsilon^{-1}\right) l} \leq \widetilde{C}\left\|A^{-1}\right\|_{\alpha} 2^{-\left(\alpha-\varepsilon_{0}(1+\varepsilon)\right) l} .
$$

Moreover, Lemma 4.5 implies that if $\lambda^{\prime} \in N^{\varepsilon}(\lambda)$, then $\lambda^{\prime} \subset \lambda_{0}$. Consequently, we have $\left|x_{\lambda^{\prime}}\right| \leq 2^{-\left(\alpha-\varepsilon_{0}(1+\varepsilon)\right) l}$ and Lemma 4.1 gives

$$
\left|\sum_{\lambda^{\prime} \in N^{\varepsilon}(\lambda)} A^{-1}\left(\lambda, \lambda^{\prime}\right) x_{\lambda^{\prime}}\right| \leq \widetilde{C}\left\|A^{-1}\right\|_{\alpha} 2^{-\left(\alpha-\varepsilon_{0}(1+\varepsilon)\right) l} .
$$

So, we get that

$$
\left|c_{\lambda}\right| \leq 2 \widetilde{C}\left\|A^{-1}\right\|_{\alpha} 2^{-\left(\alpha-\varepsilon_{0}(1+\varepsilon)\right) l}
$$


and

$$
\sup _{\lambda \in \operatorname{Cond}_{\varepsilon}\left(\lambda_{0}\right)}\left|c_{j, k}\right| \leq 2 \widetilde{C}\left\|A^{-1}\right\|_{\alpha} 2^{-\left(\alpha-\varepsilon_{0}(1+\varepsilon)\right) l} .
$$

Consequently, we have

$$
\begin{gathered}
\#\left\{\lambda_{0} \in \Lambda_{l}: \sup _{\lambda^{\prime} \subset \lambda_{0}}\left|x_{\lambda^{\prime}}\right| \leq 2^{-\left(\alpha-\varepsilon_{0}(1+\varepsilon)\right) l}\right\} \\
\leq \#\left\{\lambda_{0} \in \Lambda_{l}: \sup _{\lambda \in \operatorname{Cond}_{\varepsilon}\left(\lambda_{0}\right)}\left|c_{\lambda}\right| \leq 2 \widetilde{C}\left\|A^{-1}\right\|_{\alpha} 2^{-\left(\alpha-\varepsilon_{0}(1+\varepsilon)\right) l}\right\} .
\end{gathered}
$$

Let us choose $j_{l} \in \mathbb{N}$ such that $(1-2 \varepsilon) j_{l} \geq l+3$ and $j_{l} \leq(1+\varepsilon) l$. For every $\lambda_{0}=\lambda\left(l, k_{0}\right)$ of size $2^{-l}$, we fix $k$ such that

$$
(1-2 \varepsilon) j_{l}>l \text { and } 2^{(2 \varepsilon-1) j_{l}} \leq \frac{k}{2^{j_{l}}}-\frac{k_{0}}{2^{l}} \leq 2^{-l}-\mathbf{4} \cdot 2^{(2 \varepsilon-1) j} .
$$

Let us remark that in particular, we have $\lambda\left(j_{l}, k\right) \subset \lambda_{0}$ and therefore the $\lambda\left(j_{l}, k\right)$ are different for different $\lambda_{0}$ of size $2^{-l}$. A simple computation shows that if $\lambda^{\prime} \subset \lambda\left(j_{l}, k\right)$, then $\left(j^{\prime}, k^{\prime}\right) \in \operatorname{Cond}_{\varepsilon}\left(\lambda_{0}\right)$. It follows that

$$
\begin{aligned}
& \#\left\{\lambda_{0} \in \Lambda_{l}: \sup _{\lambda \in \operatorname{Cond}_{\varepsilon}\left(\lambda_{0}\right)}\left|c_{\lambda}\right| \leq 2 \widetilde{C}\left\|A^{-1}\right\|_{\alpha} 2^{-\left(\alpha-\varepsilon_{0}(1+\varepsilon)\right) l}\right\} \\
\leq & \#\left\{\lambda \in \Lambda_{j_{l}}: \sup _{\lambda^{\prime} \subset \lambda}\left|c_{\lambda^{\prime}}\right| \leq 2 \widetilde{C}\left\|A^{-1}\right\|_{\alpha} 2^{-\left(\alpha-\varepsilon_{0}(1+\varepsilon)\right) l}\right\} \\
\leq & \#\left\{\lambda \in \Lambda_{j_{l}}: \sup _{\lambda^{\prime} \subset \lambda}\left|c_{\lambda^{\prime}}\right| \leq 2 \widetilde{C}\left\|A^{-1}\right\|_{\alpha} 2^{-\left(\frac{\alpha}{1+\varepsilon}-\varepsilon_{0}\right) j_{l}}\right\} \\
\leq & \#\left\{\lambda \in \Lambda_{j_{l}}: \sup _{\lambda^{\prime} \subset \lambda}\left|c_{\lambda^{\prime}}\right| \leq 2^{-\left(\frac{\alpha}{1+\varepsilon}-2 \varepsilon_{0}\right) j_{l}}\right\} .
\end{aligned}
$$

So, we have obtained

(*)

$\#\left\{\lambda_{0} \in \Lambda_{l}: \sup _{\lambda^{\prime} \subset \lambda_{0}}\left|x_{\lambda^{\prime}}\right| \leq 2^{-\left(\alpha-\varepsilon_{0}(1+\varepsilon)\right) l}\right\} \leq \#\left\{\lambda \in \Lambda_{j_{l}}: \sup _{\lambda^{\prime} \subset \lambda}\left|c_{\lambda^{\prime}}\right| \leq 2^{-\left(\frac{\alpha}{1+\varepsilon}-2 \varepsilon_{0}\right) j_{l}}\right\}$.

This inequality holds for every $\varepsilon \in\left(0, \frac{1}{2}\right)$ such that $\alpha-r<\varepsilon^{-1}$ (using the corresponding $\varepsilon_{0}$ and $j_{l}$ ). Let us now consider three different cases. As usually, we denote

$$
\alpha_{\max }=\sup \left\{\alpha: \theta_{\vec{c}}^{-}(\alpha) \geq 0\right\}
$$

possibly equal to $+\infty$.

1. Assume that $\alpha \leq \alpha_{\max }<+\infty$.

Let us first fix $\delta>0$. Using the left continuity of $\theta_{\vec{c}}^{-}$, we can assume that $\varepsilon>0$ is small enough so that $\varepsilon<\delta$ and

$$
\theta_{\vec{c}}^{-}\left(\frac{\alpha}{1+\varepsilon}\right)-\theta_{\vec{c}}^{-}(\alpha) \leq \delta .
$$


From the definition of $\theta_{\vec{c}}^{-}$, we can assume that

$$
\#\left\{\lambda \in \Lambda_{j}: \sup _{\lambda^{\prime} \subset \lambda}\left|c_{\lambda^{\prime}}\right| \leq 2^{-\left(\frac{\alpha}{1+\varepsilon}-2 \varepsilon_{0}\right) j}\right\} \leq 2^{\left(\theta_{\vec{c}}^{-}\left(\frac{\alpha}{1+\varepsilon}\right)+\delta\right) j} \leq 2^{\left(\theta_{\vec{c}}^{-}(\alpha)+2 \delta\right) j}
$$

for $j \geq J$. We get from $(*)$

$$
\begin{aligned}
\#\left\{\lambda_{0} \in \Lambda_{l}: \sup _{\lambda^{\prime} \subset \lambda_{0}}\left|x_{\lambda^{\prime}}\right| \leq 2^{-\left(\alpha-\varepsilon_{0}(1+\varepsilon)\right) l}\right\} & \leq 2^{\left(\theta_{\vec{c}}^{-}(\alpha)+2 \delta\right) j_{l}} \\
& \leq 2^{\left(\theta_{\vec{c}}^{-}(\alpha)+2 \delta\right)(1+\varepsilon) l} \\
& =2^{\left(\theta_{\vec{c}}^{-}(\alpha)+2 \delta+\varepsilon \theta_{\vec{c}}^{-}(\alpha)+2 \varepsilon \delta\right) l} \\
& \leq 2^{\left(\theta_{\vec{c}}^{-}(\alpha)+5 \delta\right) l}
\end{aligned}
$$

for every $l$ large enough. It follows that $\theta_{\vec{x}}^{-}(\alpha) \leq \theta_{\vec{c}}^{-}(\alpha)$.

2. Assume that $\alpha_{\max }<+\infty$ and $\alpha>\alpha_{\max }$

This case is immediate since

$$
\#\left\{\lambda \in \Lambda_{j_{l}}: \sup _{\lambda^{\prime} \subset \lambda}\left|c_{\lambda^{\prime}}\right| \leq 2^{-\left(\frac{\alpha}{1+\varepsilon}-2 \varepsilon_{0}\right) j_{l}}\right\}=0
$$

for every $j$ large enough.

\section{Assume that $\alpha_{\max }=+\infty$.}

Let us fix $\delta>0$. Again, we assume that $\varepsilon<\delta$. From the definition of $\theta_{\vec{c}}^{-}(+\infty)$, for every $\alpha$ large enough, we have

$$
\#\left\{\lambda \in \Lambda_{j}: \sup _{\lambda^{\prime} \subset \lambda}\left|c_{\lambda^{\prime}}\right| \leq 2^{-\left(\frac{\alpha}{1+\varepsilon}-2 \varepsilon_{0}\right) j}\right\} \leq 2^{\left(\theta_{\vec{c}}^{-}(+\infty)+\delta\right) j}
$$

for infinitely many $j$. Given such a $j$, we consider $l \in \mathbb{N}$ such that $(1-2 \varepsilon) j \geq l+3$ and $j \leq(1+\varepsilon) l$. Using $(*)$, we get

$$
\begin{aligned}
\#\left\{\lambda_{0} \in \Lambda_{l}: \sup _{\lambda^{\prime} \subset \lambda_{0}}\left|x_{\lambda^{\prime}}\right| \leq 2^{-\left(\alpha-\varepsilon_{0}(1+\varepsilon)\right) l}\right\} & \leq \#\left\{\lambda \in \Lambda_{j}: \sup _{\lambda^{\prime} \subset \lambda}\left|c_{\lambda^{\prime}}\right| \leq 2^{-\left(\frac{\alpha}{1+\varepsilon}-2 \varepsilon_{0}\right) j}\right\} \\
& \leq 2^{\left(\theta_{\vec{c}}^{-}(+\infty)+\delta\right) j} \\
& \leq 2^{\left(\theta_{\vec{c}}^{-}(+\infty)+2 \delta\right)(1+\varepsilon) l} \\
& \leq 2^{\left(\theta_{\vec{c}}^{-}(+\infty)+5 \delta\right) l} .
\end{aligned}
$$

Since it holds for infinitely many $l$, this concludes the proof.

Consequently, given a function $f$, we can define its increasing (resp. decreasing) wavelet profile $\theta_{f}^{+}\left(\operatorname{resp} \theta_{f}^{-}\right)$by setting $\theta_{f}^{+}=\theta_{\vec{c}}^{+}$(resp. $\theta_{f}^{-}=\theta_{\vec{c}}^{-}$), where $\vec{c}$ is the sequence of wavelet coefficients of $f$ in a given wavelet basis in the Schwartz class. 


\section{5. $\mathcal{L}^{\nu}$ profile spaces}

We are now ready to define a new notion which is both robust and encapsulates the information supplied by the increasing and decreasing wavelet leaders profiles. In [11], the authors have investigated which ones of the results, proved in [6] in the wavelet coefficients setting, can be extended in the wavelet leaders setting. In particular, these new spaces are compared with the $\mathcal{S}^{\nu}$ spaces, previously introduced using wavelet coefficients. The main difference is that now the profile includes an increasing and a decreasing part, and is therefore more realistic for most multifractal models, for which the decreasing part can prove crucial for identification, or model selection, see [28] for instance.

The properties of the wavelet leaders profiles (see Proposition 3.3) naturally lead to the following definition: an admissible profile is a function $\nu:[0,+\infty) \rightarrow$ $\{-\infty\} \cup[0,1]$ for which there exist $0 \leq \alpha_{\min } \leq \alpha_{s} \leq \alpha_{\max }$ such that

$$
\left\{\begin{array}{l}
\nu=-\infty \text { on }\left[0, \alpha_{\min }\right) \cup\left(\alpha_{\max },+\infty\right], \\
\nu\left(\alpha_{s}\right)=1, \\
\nu \text { is increasing, right-continuous and takes values in }[0,1] \text { on }\left[\alpha_{\min }, \alpha_{s}\right], \\
\nu \text { is decreasing, left-continuous and takes values in }[0,1] \text { on }\left[\alpha_{s}, \alpha_{\max }\right], \\
\alpha \mapsto \frac{\nu(\alpha)-1}{\alpha} \text { is decreasing on }\left[\alpha_{s}, \alpha_{\max }\right] .
\end{array}\right.
$$

We also include the cases where $\alpha_{s}$ or $\alpha_{\max }$ is equal to $+\infty$ (if $\alpha_{\max }=+\infty$, the left continuity of $\nu$ is only considered on $\left.\left(\alpha_{s}, \alpha_{\max }\right)\right)$. Let us mention that in $[11,15]$, the authors have proved that for any admissible profile $\nu$ with $\alpha_{\max }<+\infty$, there is $\vec{c} \in C^{0}(\mathbb{T})$ such that

$$
\nu(\alpha)= \begin{cases}\theta_{\vec{c}}^{+}(\alpha) & \text { if } \alpha<\alpha_{s}, \\ \theta_{\vec{c}}^{-}(\alpha) & \text { if } \alpha \geq \alpha_{s},\end{cases}
$$

so Proposition 3.3 entirely characterizes the wavelet leaders profiles.

This leads naturally to the following definition, motivated by Remark 3.6.

Definition 5.1. Given an admissible profile $\nu$, the profile space $\mathcal{L}^{\nu}$ is

$$
\mathcal{L}^{\nu}:=\left\{\vec{c} \in C^{0}(\mathbb{T}): \theta_{\vec{c}}^{+}(\alpha) \leq \nu(\alpha) \text { if } \alpha<\alpha_{s} \text { and } \theta_{\vec{c}}^{-}(\alpha) \leq \nu(\alpha) \text { if } \alpha>\alpha_{s}\right\} .
$$

Remark 5.2. If a sequence $\vec{c}$ belongs to $\mathcal{L}^{\nu}$ and if $\alpha_{\max }$ is finite, then the hypothesis of Lemma 3.5 are automatically satisfied.

Remark 5.3. In general, a profile space $\mathcal{L}^{\nu}$ is not a vector space since 0 does not belong to $\mathcal{L}^{\nu}$. Nevertheless, if one considers an admissible profile such that $\nu(\alpha)=1$ for every $\alpha \geq \alpha_{s}$, it is easy to see that $\mathcal{L}^{\nu}$ is a vector space. Moreover, in this case, the definition of an admissible profile coincides with the one considered in the case of $\mathcal{S}^{\nu}$ spaces [6] (this justifies the use of the notation $\nu$ ).

The robustness of the wavelet leaders profiles directly implies the following result. 
Proposition 5.4. Let $\nu$ be an admissible profile. Then the profile space $\mathcal{L}^{\nu}$ is robust.

\section{Examples}

It follows from Propositions 4.4 and 4.6 that the increasing and decreasing wavelet leaders profiles of a uniformly Hölder function do not depend on the wavelet basis chosen in the Schwartz class. Proposition 3.4 states that they give an upper bound for the multifractal spectrum of this function. The wavelet leaders profiles are therefore a "good candidate" for a multifractal formalism based on wavelet leaders, as mentioned in Remark 3.6. In this section, we show that this formalism holds for some classical models used in signal and image processing.

\subsection{Deterministic cascades on wavelet dyadic trees}

The first model we consider is a deterministic wavelet cascade; it is the simplest case of the famous cascade models which have been introduced as turbulence models, and are also used in financial modeling.

Let us consider the binomial measure $\mu$ of parameter $p \in(0,1)$, which is the unique measure supported on $[0,1]$ such that

$$
\mu\left(\lambda_{j, k}\right)=p^{\phi(j, k)}(1-p)^{j-\phi(j, k)}
$$

where $\phi(j, k)$ is the number of 1 among the $j$ 's first coordinates in the dyadic decomposition of $k 2^{-j}$. Following a general framework proposed by Barral and Seuret in [10], let us construct the wavelet series $F_{\mu}$ by prescribing its wavelet coefficients in a given wavelet basis as follows: for every $\lambda$, we set $c_{\lambda}=\mu(\lambda)$. We will say that $F_{\mu}$ is a deterministic Bernoulli cascade of parameter $p$. Remark that the wavelet coefficients of $F_{\mu}$ are simply defined recursively by

$$
\begin{cases}c_{0,0} & =1 \\ c_{j, 2 k} & =(1-p) c_{j-1, k} \\ c_{j, 2 k+1} & =p c_{j-1, k}\end{cases}
$$

for all $j \geq 1$ and $k \in\left\{0, \ldots, 2^{j}-1\right\}$. At each scale $j \in \mathbb{N}_{0}$, we have $\left(\begin{array}{l}j \\ l\end{array}\right)$ coefficients of size $p^{l}(1-p)^{j-l}$ for $l \in\{0, \ldots, j\}$. In order to avoid trivial cases, we will assume that $p \neq 1 / 2$. We will also assume that $p<1 / 2$; the case $p>1 / 2$ is similar.

Following the seminal work [31] the study of the multifractal spectrum and of the large deviation properties of these functions and their underlying measures (and of more general models of cascades) has been the subject of numerous studies (see e.g. $[10,12,13,14,17,38]$ and references therein). In particular, the wavelet series of $F_{\mu}$ converges to a continuous function which has the following properties. 
Proposition 6.1. Let $F_{\mu}$ be a deterministic cascade of parameter $p \in\left(0, \frac{1}{2}\right)$. Then $d_{F_{\mu}}(\alpha)= \begin{cases}-\left(\beta \log _{2}(\beta)+(1-\beta) \log _{2}(1-\beta)\right) & \text { if } \alpha \in\left(-\log _{2}(1-p),-\log _{2}(p)\right), \\ 0 & \text { if } \alpha \in\left\{-\log _{2}(1-p),-\log _{2}(p)\right\}, \\ -\infty & \text { otherwise, }\end{cases}$ where

$$
\beta=\frac{\alpha+\log _{2}(1-p)}{\log _{2}(1-p)-\log _{2}(p)} .
$$

Moreover, if $\vec{c}$ denotes the sequence of wavelet coefficients given by (**), then $\rho_{\vec{c}}=d_{F_{\mu}}$.

Remark that the function $d_{F_{\mu}}$ has a unique maximum realized at the point $\alpha_{s}=-\frac{1}{2} \log _{2}((1-p) p)$. We set

$$
\theta_{F_{\mu}}(\alpha)= \begin{cases}\theta_{F_{\mu}}^{+}(\alpha) & \text { if } \alpha<\alpha_{s} \\ \theta_{F_{\mu}}^{-}(\alpha) & \text { if } \alpha \geq \alpha_{s}\end{cases}
$$

Note that the second assertion of Proposition 6.1 holds in the particular case where the analyzing wavelet is the same as the synthesis one. However, since the definition of the wavelet leaders profile is robust, the following corollary holds even if the the analyzing and synthesis wavelets differ.

Corollary 6.2. Let $p \in(0,1)$ and $\alpha_{s}=-\frac{1}{2} \log _{2}((1-p) p)$. Let $F_{\mu}$ be is a deterministic cascade of parameter $p$, then

$$
\theta_{F_{\mu}}=d_{F_{\mu}}
$$

Proof. The result follows directly from Proposition 6.1 and Lemma 3.5.

\subsection{Thresholded Wavelet Series}

Let $f$ be a function whose wavelet coefficients in a wavelet basis $\left(\psi_{\lambda}\right)_{\lambda \in \Lambda}$ are given by $\vec{c}$ and let $\gamma>0$. Following [37], the wavelet series $f^{t}$ defined by

$$
f^{t}=\sum_{j \in \mathbb{N}_{0}} \sum_{\lambda \in \Lambda_{j}} c_{\lambda}^{t} \psi_{\lambda} \text { where } c_{\lambda}^{t}=c_{\lambda} \mathbf{1}_{|\cdot| \geq 2^{-\gamma j}}\left(c_{\lambda}\right) \quad \forall \lambda \in \Lambda_{j},
$$

is said to be obtained from $f$ after a threshold of order $\gamma$. This method was introduced by Seuret in order to create functions with oscillating singularities. They also display non-concave multifractal spectra, as stated by the following proposition [37].

Proposition 6.3. Let $F_{\mu}$ be a deterministic Bernoulli cascade of parameter $p \in$ $\left(0, \frac{1}{2}\right)$. Let $\omega_{t}:\left[\gamma,-\log _{2}(p)\right] \rightarrow(0,+\infty)$ be the increasing function

$$
u \mapsto \gamma \frac{u+\log _{2}(1-p)}{\gamma+\log _{2}(1-p)},
$$




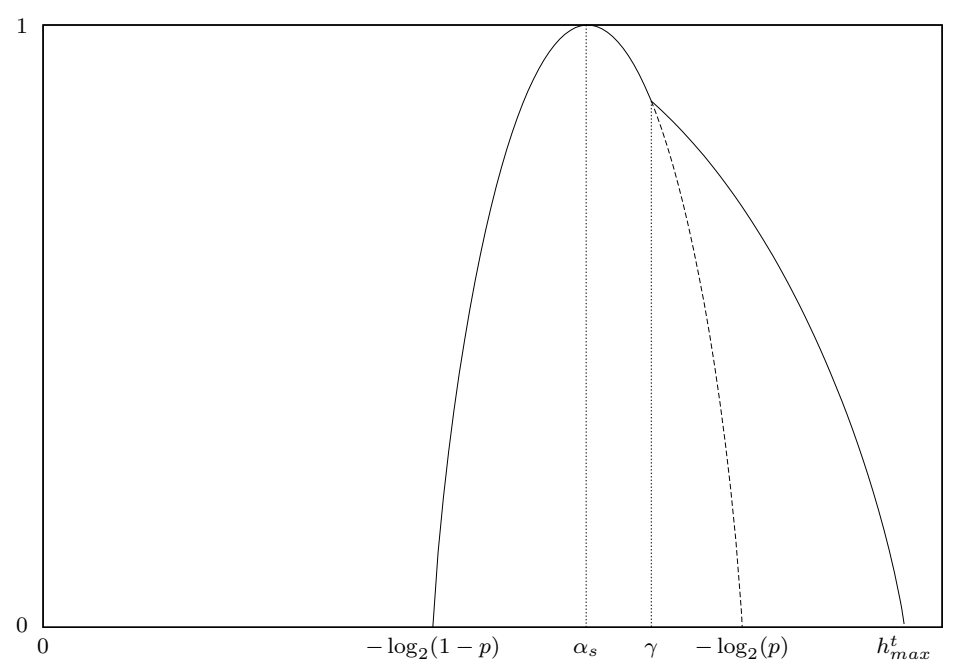

Figure 2: Multifractal spectrum of $F_{\mu}^{t}$ for $p=0.4$ and $\gamma=1.2$.

and let $h_{\max }^{t}=\omega_{t}\left(-\log _{2}(p)\right)$. If $\gamma \in\left[-\log _{2}(1-p),-\log _{2}(p)\right]$, the multifractal spectrum of $F_{\mu}^{t}$ takes values in $\left[-\log _{2}(1-p), h_{\max }^{t}\right]$ and is equal to

$$
d_{F_{\mu}^{t}}(h)= \begin{cases}d_{f}(h) & \text { if } h \in\left[-\log _{2}(1-p), \gamma\right], \\ d_{f}\left(\omega_{t}^{-1}(h)\right) & \text { if } h \in\left(\gamma, h_{\max }^{t}\right]\end{cases}
$$

As done previously, let us denote by

$$
\alpha_{s}=-\frac{1}{2} \log _{2}((1-p) p),
$$

the point at which $d_{F_{\mu}}$ is maximum. If $\gamma>\alpha_{s}$, the spectrum is non concave in its decreasing part (see [37] and Figure 2) and therefore, all multifractal formalisms proposed up to now fail for its estimation. We consider the function $\theta_{F_{\mu}^{t}}$ defined by

$$
\theta_{F_{\mu}^{t}}(\alpha)= \begin{cases}\theta_{F_{\mu}^{t}}^{+}(\alpha) & \text { if } \alpha<\alpha_{s} \\ \theta_{F_{\mu}^{t}}^{-}(\alpha) & \text { if } \alpha \geq \alpha_{s} .\end{cases}
$$

Let us show that the computation of this wavelet profile of $F_{\mu}^{t}$ leads to the correct spectrum.

Proposition 6.4. Let $F_{\mu}$ be a deterministic Bernoulli cascade of parameter $p \in$ $\left(0, \frac{1}{2}\right)$ and let $F_{\mu}^{t}$ be the wavelet series obtained from $F_{\mu}$ after a threshold of order $\gamma>\alpha_{s}$ where $\alpha_{s}=-\frac{1}{2} \log _{2}((1-p) p)$. Then we have $\theta_{F_{\mu}^{t}}=d_{F_{\mu}^{t}}$.

Proof. From Proposition 3.4, it suffices to show that $\theta_{F_{\mu}^{t}} \leq d_{F_{\mu}^{t}}$. Let us denote by $e_{\lambda}$ the restricted wavelet leaders of $F_{\mu}$ and by $e_{\lambda}^{t}$ the restricted wavelet leaders of $F_{\mu}^{t}$. 
First assume that $\alpha<\alpha_{s}$. We clearly have $\theta_{F_{\mu}^{t}}(\alpha) \leq \theta_{F_{\mu}}^{+}(\alpha)$ and therefore, $\theta_{F_{\mu}^{t}}(\alpha) \leq d_{F_{\mu}^{t}}(\alpha)$ using Corollary 6.2.

Secondly, if $\alpha \in\left[\alpha_{s}, \gamma\right]$, we have

$$
\#\left\{\lambda \in \Lambda_{j}: e_{\lambda}^{t} \leq 2^{-(\alpha-\varepsilon) j}\right\} \leq \#\left\{\lambda \in \Lambda_{j}: e_{\lambda} \leq 2^{-(\alpha-\varepsilon) j}\right\} .
$$

Indeed, if $e_{\lambda}>2^{-(\alpha-\varepsilon) j}$, then $c_{\lambda}=e_{\lambda} \geq 2^{-\gamma j}$. It follows that $c_{\lambda}^{t}=c_{\lambda}=e_{\lambda}$ and $e_{\lambda}^{t}=c_{\lambda}$. Therefore, $e_{\lambda}^{t}>2^{-(\alpha-\varepsilon) j}$ and $\theta_{F_{\mu}^{t}}(\alpha) \leq \theta_{F_{\mu}}^{-}(\alpha)=d_{f}(\alpha)$.

Finally, assume that $\alpha>\gamma$. Remark that if $\lambda \in \Lambda_{j}$ is such that $e_{\lambda}^{t} \leq 2^{-(\alpha-\varepsilon) j}$ with $\alpha-\varepsilon>\gamma$, then $e_{\lambda}^{t}=p^{l}(1-p)^{\left\lfloor C_{\gamma} l\right\rfloor+1-j}$, where

$$
C_{\gamma}=\frac{\log _{2}(1-p)-\log _{2}(p)}{\gamma+\log _{2}(1-p)}
$$

and $\beta_{\varepsilon, j} \leq l \leq j$, where

$\beta_{\varepsilon, j}=\frac{\log _{2}(1-p)+(\alpha-\varepsilon) j}{\left(1-C_{\gamma}\right) \log _{2}(1-p)-\log _{2}(p)}=\frac{\left(\log _{2}(1-p)+(\alpha-\varepsilon) j\right)\left(\gamma+\log _{2}(1-p)\right)}{\gamma\left(\log _{2}(1-p)-\log _{2}(p)\right)}$.

Moreover, since $\alpha>\gamma>\alpha_{s}$, such a $l$ is bigger than $j / 2$ for $j$ large enough. Therefore,

$$
\#\left\{\lambda \in \Lambda_{j}: e_{\lambda}^{t} \leq 2^{-(\alpha-\varepsilon) j}\right\} \leq \sum_{l=\left\lfloor\beta_{\varepsilon, j}\right\rfloor}^{j}\left(\begin{array}{l}
j \\
l
\end{array}\right) \leq j\left(\begin{array}{c}
j \\
\left\lfloor\beta_{\varepsilon, j}\right\rfloor
\end{array}\right)
$$

and it follows that

$$
\begin{aligned}
& \frac{\log \#\left\{\lambda \in \Lambda_{j}: e_{\lambda}^{t} \leq 2^{-(\alpha-\varepsilon) j}\right\}}{\log 2^{j}} \\
\leq & \frac{1}{j} \log _{2}\left(j\left(\begin{array}{c}
j \\
\left\lfloor\beta_{\varepsilon, j}\right\rfloor
\end{array}\right)\right) \\
\sim & \frac{1}{j} \log _{2}\left(\frac{j \sqrt{2 \pi j}\left(\frac{j}{e}\right)^{j}}{\sqrt{2 \pi\left\lfloor\beta_{\varepsilon, j}\right\rfloor}\left(\frac{\left\lfloor\beta_{\varepsilon, j}\right\rfloor}{e}\right)^{\left\lfloor\beta_{\varepsilon, j}\right\rfloor} \sqrt{2 \pi\left(j-\left\lfloor\beta_{\varepsilon, j}\right\rfloor\right)}\left(\frac{j-\left\lfloor\beta_{\varepsilon, j}\right\rfloor}{e}\right)^{j-\left\lfloor\beta_{\varepsilon, j}\right\rfloor}}\right)
\end{aligned}
$$

where we have used Stirling formula. Moreover,

$$
\begin{aligned}
& \frac{1}{j} \log _{2}\left(\frac{j \sqrt{2 \pi j}\left(\frac{j}{e}\right)^{j}}{\sqrt{2 \pi\left\lfloor\beta_{\varepsilon, j}\right\rfloor}\left(\frac{\left\lfloor\beta_{\varepsilon, j}\right\rfloor}{e}\right)^{\left\lfloor\beta_{\varepsilon, j}\right\rfloor} \sqrt{2 \pi\left(j-\left\lfloor\beta_{\varepsilon, j}\right\rfloor\right)}\left(\frac{j-\left\lfloor\beta_{\varepsilon, j}\right\rfloor}{e}\right)^{j-\left\lfloor\beta_{\varepsilon, j}\right\rfloor}}\right) \\
= & \frac{1}{j} \log _{2}\left(\frac{j \sqrt{j}}{\left(\frac{\left\lfloor\beta_{\varepsilon, j}\right\rfloor}{j}\right)^{\left\lfloor\beta_{\varepsilon, j}\right\rfloor} \sqrt{2 \pi\left\lfloor\beta_{\varepsilon, j}\right\rfloor\left(j-\left\lfloor\beta_{\varepsilon, j}\right\rfloor\right)}\left(\frac{j-\left\lfloor\beta_{\varepsilon, j}\right\rfloor}{j}\right)^{j-\left\lfloor\beta_{\varepsilon, j}\right\rfloor}}\right) \\
= & \frac{1}{j} \log _{2}\left(\frac{\sqrt{j}}{\left(\frac{\left\lfloor\beta_{\varepsilon, j}\right\rfloor}{j}\right)^{\left\lfloor\beta_{\varepsilon, j}\right\rfloor} \sqrt{2 \pi \frac{\left\lfloor\beta_{\varepsilon, j}\right\rfloor}{j}\left(1-\frac{\left\lfloor\beta_{\varepsilon, j}\right\rfloor}{j}\right)}\left(1-\frac{\left\lfloor\beta_{\varepsilon, j}\right\rfloor}{j}\right)^{j-\left\lfloor\beta_{\varepsilon, j}\right\rfloor}}\right) \\
= & \frac{1}{j} \log _{2}\left(\frac{\sqrt{j}}{\sqrt{2 \pi \frac{\left\lfloor\beta_{\varepsilon, j}\right\rfloor}{j}\left(1-\frac{\left\lfloor\beta_{\varepsilon, j}\right\rfloor}{j}\right)}}\right)-\log _{2}\left(\frac{\left\lfloor\beta_{\varepsilon, j}\right\rfloor}{j} \frac{\left\lfloor\beta_{\varepsilon, j}\right\rfloor}{j}\left(1-\frac{\left\lfloor\beta_{\varepsilon, j}\right\rfloor}{j}\right)^{1-\frac{\left\lfloor\beta_{\varepsilon, j}\right\rfloor}{j}}\right) .
\end{aligned}
$$


If we compute the limit for $j \rightarrow+\infty$ and $\varepsilon \rightarrow 0^{+}$, we get that

$$
\theta_{F_{\mu}^{t}}(\alpha) \leq-\left(\beta \log _{2}(\beta)+(1-\beta) \log _{2}(1-\beta)\right)
$$

with

$$
\beta=\lim _{\varepsilon \rightarrow 0^{+}} \lim _{j \rightarrow+\infty} \frac{\beta_{\varepsilon, j}}{j}=\alpha \frac{\gamma+\log _{2}(1-p)}{\gamma\left(\log _{2}(1-p)-\log _{2}(p)\right)} .
$$

The conclusion follows from Proposition 6.3.

Acknowledgement. The authors thank T. Kleyntssens from the University of Liège for the realization of Figure 1 and Figure 2, and the anonymous referee for his suggestions, which greatly improved the redaction of this paper.

\section{References}

[1] P. Abry, S. Jaffard, and H. Wendt. A bridge between geometric measure theory and signal processing: Multifractal analysis. Operator-Related Function Theory and Time-Frequency Analysis, The Abel Symposium 2012, Abel Symposia Vol. 9, Springer K. Grchenig, Y. Lyubarskii, K. Seip, Kristian Eds., pages 1-56, 2015.

[2] P. Abry, S. Jaffard, and H. Wendt. Irregularities and scaling in signal and image processing: Multifractal analysis. Benoit Mandelbrot: A Life in Many Dimensions, M. Frame Ed., World scientific publishing, pages 31-116, 2015.

[3] A. Arneodo, E. Bacry, and J.F. Muzy. The thermodynamics of fractals revisited with wavelets. Phys. A., 213:232-275., 1995.

[4] J.M. Aubry and F. Bastin. A walk from multifractal analysis to functional analysis with $S^{\nu}$ spaces, and back. In Recent Developments in Fractals and Related Fields, pages 93-106. Springer, 2010.

[5] J.M. Aubry, F. Bastin, and S. Dispa. Prevalence of multifractal functions in $S^{\nu}$ spaces. J. Fourier Anal. Appl., 13(2):175-185., 2007.

[6] J.M. Aubry, F. Bastin, S. Dispa, and S. Jaffard. Topological properties of the sequence spaces $S^{\nu}$. J. Fourier Anal. Appl., 321(1):364-387, 2006.

[7] J.M. Aubry and S. Jaffard. Random wavelet series. Comm. Math. Phys., 227(3):483$514,2002$.

[8] E. Bacry, J. Delour, and J.F. Muzy. Multifractal random walk. Phys. Rev. E, 64(2):026103, 2001.

[9] J. Barral and P. Gonçalves. On the estimation of the large deviations spectrum. Journal of Statistical Physics, 144(6):1256-1283, 2011.

[10] J. Barral and S. Seuret. From multifractal measures to multifractal wavelet series. J. Four. Anal. Appl., 11(5):589-614, 2005.

[11] F. Bastin, C. Esser, and L. Simons. Topology on new sequence spaces defined with wavelet leaders. Journal of Mathematical Analysis and Applications, 431(1):317-341, 2015.

[12] G. Brown, G. Michon, and J. Peyrière. On the multifractal analysis of measures. J. Statist. Phys., 66:775-790, 1992. 
[13] R. Cawlet and R. D. Mauldin. Multifractal decompositions of Moran fractals. Adv. in Math., 92:196-236, 1992.

[14] H. G. Eggleston. The fractional dimension of a set defined by decimal properties. Q. J. Math. Oxford, 20:31-46, 1949.

[15] C. Esser. Regularity of functions: genericity and multifractal analysis. $\mathrm{PhD}$ thesis, Université de Liège, 2014 (the document can be loaded at http://orbi.ulg.ac.be/handle/2268/174112).

[16] C. Esser, T. Kleyntssens, and S. Nicolay. A multifractal formalism for non-concave and non-increasing spectra: the leaders profile method. Submitted for publication.

[17] K. Falconer. Fractal Geometry: Mathematical Foundation and Applications. John Wiley \& Sons, 1990.

[18] A. Fraysse and S. Jaffard. How smooth is almost every function in a Sobolev space? Revista Matematica Iberoamericana, 22(2):663-682, 2006.

[19] S. Jaffard. Multifractal formalism for functions. SIAM J. Math. Anal., 28:441-460., 1997.

[20] S. Jaffard. The multifractal nature of Lévy processes. Proba. Theo. Related Fields, 114(2):207-227, 1999 .

[21] S. Jaffard. On the Frisch-Parisi conjecture. J. Math. Pures Appl., 79(6):525-552., 2000 .

[22] S. Jaffard. Beyond Besov Spaces Part 1: Distributions of Wavelet Coefficients. J. Four. Anal. Appl., 10(3):221-246, 2004.

[23] S. Jaffard. Wavelet Techniques in Multifractal Analysis. Fractal Geometry and Applications : A Jubilee of Benoit Mandelbrot, M. Lapidus et M. van Frankenhuijsen Eds., Proceedings of Symposia in Pure Mathematics, 72(2):91-151, 2004.

[24] S. Jaffard. Beyond Besov Spaces Part 2: Oscillation Spaces. Constr. Approx., 21:2961,2005

[25] S. Jaffard, P. Abry, S.G. Roux, B. Vedel, and H. Wendt. The contribution of wavelets in multifractal analysis, pages 51-98. Series in contemporary applied mathematics. World scientific publishing, 2010.

[26] S. Jaffard, B. Lashermes, and P. Abry. Wavelet Leaders in Multifractal Analysis. Wavelet analysis and applications, Appl. Numer. Harmon. Anal., pages 201-246, 2006.

[27] T. Kleyntssens, C. Esser, and S. Nicolay. A multifractal formalism based on the $S^{\nu}$ spaces: from theory to practice. Submitted for publication.

[28] B. Lashermes, S.G. Roux, P. Abry, and S. Jaffard. Comprehensive multifractal analysis of turbulent velocity using the wavelet leaders. European Physical Journal $B, 61(2): 201-215,2008$.

[29] P.G. Lemarié and Y. Meyer. Ondelettes et bases hilbertiennes. Revista Math.Iberoamericana, 1:1-18, 1986.

[30] S. Mallat and W. L. Hwang. Singularity detection and processing with wavelets. IEEE Transactions on Information Theory, 38(2):617643, 1992.

[31] B. Mandelbrot. Multiplications aléatoires itérées et distributions invariantes par moyennes pondérées. C.R. Acad. Sci. Paris, 278:289-292 and 355-358, 1974. 
[32] P. Mannersalo, I. Norros, and R. Riedi. Multifractal analysis of infinite products of stationary jump processes. J. Probability and Statistics, ID 807491:1-26, 2010.

[33] Y. Meyer. Ondelettes et oprateurs. Hermann, 1990.

[34] J. F. Muzy, E. Bacry, and A. Arneodo. Multifractal formalism for fractal signals: The structure-function approach versus the wavelet-transform modulus-maxima method. Phys. Rev. E, 47(2):875, 1993.

[35] J. F. Muzy, E. Bacry, and A. Arneodo. The multifractal formalism revisited with wavelets. Int. J. Bifurcation and Chaos, 4:245-302, 1994.

[36] G. Parisi and U. Frisch. Fully developed turbulence and intermittency. In M. Ghil, R. Benzi, and G. Parisi, editors, Turbulence and Predictability in geophysical Fluid Dynamics and Climate Dynamics, Proc. of Int. School, page 84, Amsterdam, 1985. North-Holland.

[37] S. Seuret. Detecting and creating oscillations using multifractal methods. Math. Nachr., 279(11):1195-1211, 2006.

[38] T. Tell. Fractals, multifractals, and thermodynamics. Z. Naturforsch. A, 43:1154$1174,1988$.

Received ??

F. BAstin: University of Liège, Institute of Mathematics, B37, 4000 Liège, Belgium

E-mail: F.Bastin@ulg.ac.be

C. Esser: University of Liège, Institute of Mathematics, B37, 4000 Liège, Belgium

E-mail: Celine.Esser@ulg.ac.be

S. JAFfard: University Paris-Est - Laboratoire d'Analyse et de Mathématiques Appliquées (UMR 8050) UPEM, UPEC, CNRS, F-94010, Créteil, France

E-mail: stephane.jaffard@u-pec.fr

C. Esser is supported by a grant of Research Fellow from the Fonds National de la Recherche Scientifique (FNRS) 